\title{
Exploring scalar-photon interactions in energetic astrophysical events.
}

\author{
Ankur Chaubey $^{a}$, Manoj K. Jaiswal ${ }^{a}$ and Avijit K. Ganguly ${ }^{a * \dagger}$ \\ a) Institute of Science, Department of Physics, Banaras Hindu University, Varanasi- 221005, INDIA.
}

December 29, 2020

\begin{abstract}
Scalar fields like dilaton appear in quantum field theory (QFT) due to scale symmetry breaking. Their appeal also extends to modified theories of gravity, like $F(R)$ gravity, Horva Lifshitz gravity etc. In unified theories they make their appearance through compactification of the extra dimension. Apart from resolving the issues of compactification scale and size, the particles of their fields can also turn out to be excellent candidate to solve the dark energy (DE) and dark matter (DM) problem of the universe. In this work we study their mixing dynamics with photons in a magnetized media, by incorporating the effect of parity violating part of the photon polarization tensor, evaluated in a finite density magnetized media. This piece, though in general is odd in the external magnetic field strength $e B$; in this work we however have retained terms to $O(e B)$. We are able to demonstrate in this work that, in magnetized medium a dilatonic scalar field $(\phi)$ can excite the two transverse degrees of freedom (DOF) of the photons. One due to direct coupling and the other indirectly through the parity violating term originating due to magnetized medium effects. This results in the mixing dynamics being governed by, $3 \times 3$ mixing matrices. This mixing results in making the underlying media optically active. In this work we focus on the spectro-polarimetric imprints of these particles, on the spectra of the electromagnetic (EM) fields of Gamma Ray Bursters (GRB). Focusing on a range of parameters (i.e., magnetic field strength, plasma frequency $\left(\omega_{p}\right)$, size of the magnetized volume, coupling strength to photons and their mass) we make an attempt to point out how space-borne detectors should be designed to optimise their detection possibility.
\end{abstract}

\section{Introduction}

The study of scale symmetry and it's consequences on the dynamics of particles has drawn the attention for some time now. The particles appearing as Goldstone bosons of a spontaneously broken scale symmetry (termed dilaton, $\phi(x)$ ) [1,2, have emerged from studies in QFT. There are many theories those predict the existence of dilatons. Apart from QFT, they appear in higher dimensional unified theories, for instance, in five dimensional Kaluza-Klein theory, they appear as the five-five component of the five dimensional metric, formulated to unify gravity with electromagnetism. In string and super-string theory they appear from compactification of the extra dimensions and are called string dilaton or modouli [3,4.

*e-mail addresses: avijitk@hotmail.com

${ }^{\dagger}$ corresponding author 
In some scale invariant extensions of standard model, they are made to communicate with the standard model sector via an underlying conformal sector, where they acquire mass due to breaking of the conformal in-variance [5, 6]. This physics of these models are phenomenologically rich with predicting power that can be tested in collider based experiments. On the other hand dilatons of unified theories, acquire their masses from the curvature of the extra dimension.

They couple to the standard model fields by the trace of their energy momentum tensor $T_{\nu}^{\mu}$, associated with the anomalous divergence of the dilaton 4-current 1 . Due to this, dilatons may induce other observable signatures, like dilatonic fifth force: [7-11]; bending of light [12], violation of equivalence principle [13], decay into two photons and optical activity [14 18] in external magnetic field $(B)$. When the last two phenomena - that is decay of $\phi$ into two mass-less spin one photons and optical activity-follow from the interaction Lagrangian,

$$
L_{i n t}=-\frac{1}{4 M} \phi F^{\mu \nu} F_{\mu \nu}
$$

In equation (1.1) $F^{\mu \nu}$ is the usual field strength tensor for EM field. And $M$ is the symmetry breaking scale related to the inverse of the coupling constant $g_{\phi \gamma \gamma}$, between the quanta of scalar $(\phi)$ and photon $(\gamma)$ fields. Equation (1.1) leads to their lifetime $\tau_{\phi}$ against decay to two photons $\phi \rightarrow \gamma \gamma$, given by $\tau_{\phi} \sim \frac{1}{g_{\phi \gamma \gamma}^{2} m_{\phi}^{3}}[1]$. If the life time of these particles for some values of $g_{\phi \gamma \gamma}$ and $m_{\phi}$, turn out to be comparable to the age of the universe, then the particles of the field $\phi$ will turn out to be excellent candidates for DM. Thus, simultaneously solving the two out standing problems of contemporary physics. There are other particles those produce similar signals can be found in [19 46] but we will not discuss those in this work.

Given the state of our current understanding that, about $27 \%$ of the total matter-energy density is in the form of DM, it is possible to find what percentage of the total DM density is composed of $\phi$, at some epoch $t$, as cosmological relic density $\rho(t)$, from [47]:

$$
\rho(t)=\rho_{d}\left(\frac{\eta_{d}}{\eta}\right)^{3} e^{-\frac{t}{\tau_{\phi}}}=\frac{\zeta(3)}{\pi^{2}} \frac{g^{*}(t)}{g_{* d}} T^{3}(t) e^{-\frac{t}{\tau_{\phi}}} .
$$

In equation (1.2) $\rho_{d}$ corresponds to the density and $\eta_{d}$ the magnitude of the scale factor of FriedmanRobertson-Walker metric. And $g_{* d}$ along with $g^{*}(t)$ are the number of DOF available at the time of decoupling and the same at the epoch t, respectively. The last line in equation (1.2) has been obtained demanding entropy conservation in the co-moving volume of the universe, from the time of decoupling to the epoch $t$. Since the same depends crucially on the life-time $\tau_{\phi}$, that in-turn depends on coupling constant $g_{\phi \gamma \gamma}=\frac{1}{4 M}$ and the scalar mass $m_{\phi}$ - therefore the estimations of them are of utmost importance. In this study we focus on their estimation from the EM signals originating due to the energetic activities taking place in far away magnetized astrophysical objects. To that end, we have taken a spectropolarimetric route in this paper, to estimate the parameters (mass and coupling constant) associated with these particles (dilatonic scalars) by studying their mixing dynamics in presence of magnetized plasma present in the GRB environments. We also indicate how such (spectro-polarimetric) analysis can be used to design the space-borne gamma-ray or X-ray detectors - optimally - to detect dilaton signatures through EM signals coming from GRB .

At this juncture we would like to digress a little, so as to pay attention to other possible physical sources those may contribute to the polarization of electromagnetic field, coming from far away sources. One of the possible sources that can contribute the polarization of electromagnetic beam is contribution from magnetized medium and the other can be coming due to the presence of pseudoscalar particles like axions or majorons, those couple to photons through mass dimension-5 operators Rotation of the plane

\footnotetext{
${ }^{1}$ The nonzero anomalous divergence, even for mass-less particles may realized due to scaling violation through radiative corrections
} 
of polarization of light due to magnetized medium, that is referred usually in literature as Faraday effect, takes place in a material medium having nonzero chemical potential, and a magnetic field $B$ when the magnetic field is oriented along the direction of propagation of the photon $k$. On the other hand the effect due to the scalar dilaton or pseudoscalar field, takes place, when the component of the magnetic field, is perpendicular to the wave vector $k$. So these two effects can be distinguished from each other by making the external magnetic field parallel or perpendicular to the propagation direction of the photons. It is also worth noting that, rate of rotation for plane of polarization for Faraday effect is inversely proportional to the square of the energy $\omega$ of the photons of light. Hence the same can also serve as a distinct feature to identify magnetized matter induced polarization effect from dilatons or axions. Moreover the degree of circular polarization associated with the beam of light passing through magnetized medium turns out to be zero. The details of these can be found in the Appendix A.

Now coming to the issue of distinguishing scalars(dilatons) from pseudoscalar axions, it should be noted that in magnetized vacuum, dilaton mixes with polarized light having plane of polarization oriented along the magnetic field and axion mixes with light having plane of polarization orthogonal to the magnetic field. This simple picture however gets complicated with the incorporation of magnetized matter effects. We will come back to this issue in a separate publication. Having taken these extradilaton sources, contributing to polarization of light from astrophysical sources, we turn our attention to the investigations carried out in this work.

During the course of this investigation, we have achieved few new things. They include the following (i) a better understanding of the unique nature of the set of basis vectors and the form-factors, those appear in the description of the gauge fields (i.e., of the photons), for a system in an external magnetic field $e B$, and magnetized media. We provide the transformation properties of the EM form-factors and other factors under Charge conjugation $\mathbf{C}$, Parity $\mathbf{P}$ and Time reversal $\mathbf{T}$ and use them to justify the coupling between the different DOF available to the system, leading to a $3 \times 3$ mixing matrix; (ii) We next provide the analytical route to diagonalise this matrix exactly, using an unitary similarity transformation. (iii) The resulting equations of motion obtained thereby are also exact. (iv) The numerical estimates of the Stokes parameters obtained from the numerical estimates of the form-factors, thus are also without any approximations.

The organisation of this document is as follows: In section two we introduce the details, about properties of the gauge fields (GF), the form-factors those describe them (GF) and their transformation properties under $\mathbf{C}, \mathbf{P}$ and $\mathbf{T}$. This is followed by the description of the photon polarization tensor in a magnetized medium in section three, called inclusion of matter effects. In section four we move on to analysing the equations of motion for $(\gamma-\phi)$ interacting system in a magnetized medium and demonstrate that the mixing matrix for $\phi F_{\mu \nu} F^{\mu \nu}$ interaction, turns out to be $3 \times 3$ instead of $2 \times 2$, that is usually encountered in magnetized vacuum, or unmagnetized plasma. We discuss the exact analytic diagonalization of the same in the following subsection. Subsequently we justify the same from discrete symmetry point of view. The solutions of the field equations followed by construction of the Stokes parameters is obtained in section five. In section six we introduce a typical GRB model and its environment that being used in this analysis and the polarization signals one would get from the same, for some bench mark values of $g_{\phi \gamma \gamma}$ and $m_{\phi}$, the geometry of the GRB fireball and plasma frequency. The possible EM signatures of dilaton interaction from such environments is presented in section seven. Section eight houses a discussion on the relevance of our analysis to space-borne detectors. In section nine, we conclude by providing an outlook for possible future directions of investigation. And lastly, we have provided an appendix that deals with the details of polarization evaluation in a magnetized media. Few important details regarding discrete symmetry transformations and their effects on equations of motion can be found in the supplementary materials in a separate work, titled: "Supplementary materials for Exploring scalar-photon interactions in energetic astrophysical events." 


\section{Electromagnetic form-factors for $A^{\nu}(k)$}

In the standard formulation of the massless abelian gauge theory, that describes the dynamics of photons, the action is written as,

$$
S=-\frac{1}{4} \int F^{\mu \nu} F_{\mu \nu} d^{4} x
$$

where in equation (2.1), the field strength tensor, $F^{\mu \nu}=\partial^{\mu} A^{\nu}(x)-\partial^{\nu} A^{\mu}(x)$, and $A^{\nu}(x)$ defines the gauge potentials having four DOF. The dynamics of these fields in vacuum are described by two transverse \pm 1 helicity states.

In contrast to vacuum, photons in a medium, acquire one additional DOF, (the third) longitudinal $\mathrm{DOF}$ - in addition to the two (existing) transverse degrees of freedom. In a situation like this, if there exists an external magnetic field $B$ too, then the gauge fields $A^{\nu}(k)$ corresponding to the in medium photons, can be expressed (in momentum space), in terms of four EM form-factors: $A_{\|}(k), A_{\perp}(k), A_{L}(k), A_{g f}(k)$ and four orthonormal four vectors $\left(b^{(1) \nu}, I^{\nu}, \tilde{u}^{\nu}, k^{\nu}\right)$ constructed out of the available 4 -vectors and tensors for the system (in hand). They are given by :

$$
A^{\nu}(k)=A_{\|}(k) N_{1} b^{(1) \nu}+A_{\perp}(k) N_{2} I^{\nu}+A_{L}(k) N_{L} \tilde{u}^{\nu}+N_{k} A_{g f}(k) k^{\nu} .
$$

Here, $N_{i}$ s are the normalisation constants. Rewriting $A^{\nu}(k)$ in terms of unit vectors; $\hat{b}^{(1) \nu}=N_{1} b^{(1) \nu}$, $\hat{I}^{\nu}=N_{2} I^{\nu}, \hat{\tilde{u}}^{\nu}=N_{L} b^{(1) \nu}$ and $\hat{k}^{\nu}=N_{k} k^{\nu}$ we can rewrite equation (S64) in the following form,

$$
A^{\nu}(k)=A_{\|}(k) \hat{b}^{(1) \nu}+A_{\perp}(k) \hat{I}^{\nu}+A_{L}(k) \hat{\tilde{u}}^{\nu}+A_{g f}(k) \hat{k}^{\nu} .
$$

The vectors, introduced in equation (2.3), are defined as,

$$
\begin{aligned}
& \hat{b}^{(1) \nu}=N_{1} k_{\mu} \bar{F}^{\mu \nu}, \hat{I}^{\nu}=N_{2}\left(b^{(2) \nu}-\frac{\left(\tilde{u}^{\mu} b_{\mu}^{(2)}\right)}{\tilde{u}^{2}} \tilde{u}^{\nu}\right), \hat{\tilde{u}}^{\nu}=N_{L}\left(g^{\mu \nu}-\frac{k^{\mu} k^{\nu}}{k^{2}}\right) u_{\mu}, \\
& b^{(2) \nu}=k_{\mu} \tilde{\bar{F}}^{\mu \nu}, \tilde{\bar{F}}^{\mu \nu}=\frac{1}{2} \epsilon^{\mu \nu \lambda \rho} \bar{F}_{\lambda \rho} .
\end{aligned}
$$

The normalisation constants, $N_{1}, N_{2}, N_{L}$ and $N_{k}$ in equation (S64) are given by,

$$
N_{1}=\frac{1}{\sqrt{-b_{\mu}^{(1)} b^{(1) \mu}}}=\frac{1}{K_{\perp} B}, N_{2}=\frac{1}{\sqrt{-I_{\mu} I^{\mu}}}=\frac{K}{\omega K_{\perp} B}, \quad N_{L}=\frac{1}{\sqrt{-\tilde{u}_{\mu} \tilde{u}^{\mu}}}=\frac{k^{2}}{|K|}, \text { and } N_{k}=\frac{1}{\sqrt{-k^{2}}}
$$

where $K_{\perp}=\left(k_{1}^{2}+k_{2}^{2}\right)^{\frac{1}{2}}$.

\subsection{Degrees of freedom}

In field theory, medium effects are incorporated into a system by adding a self energy corrected effective Lagrangian to the tree level Lagrangian. For electromagnetic theory, this term, in momentum space has the form $A^{\mu}(-k) \Pi_{\mu \nu}(k, T, \mu) A^{\nu}(k)$; when, $\Pi_{\mu \nu}(k)$, the polarization tensor, can be expressed in terms of transverse and longitudinal form factors, $\Pi_{T}(k, T, \mu)$ and $\Pi_{L}(k, T, \mu)$ as,

$$
\Pi_{\mu \nu}(k, T, \mu)=\Pi_{T}(k, T, \mu)\left[R_{\mu \nu}-Q_{\mu \nu}\right]+\Pi_{L}(k, T, \mu) Q_{\mu \nu} .
$$

These form factors happen to be functions of finite temperature (T), finite chemical potential $(\mu)$ and scalars made out of photon four vector $k^{\mu}$ and centre of mass four velocity of the medium $u^{\mu}$ individually, 
or as a combination such as $(k . u)$. Tensors $R_{\mu \nu}$ and $Q_{\mu \nu}$ are the transverse and longitudinal projection operators, constructed using the momentum and centre of mass velocity four vector $u^{\mu}$ as,

$$
R_{\mu \nu}=\tilde{g}_{\mu \nu}, Q_{\mu \nu}=\frac{\tilde{u}_{\mu} \tilde{u}_{\nu}}{\tilde{u}_{\mu} \tilde{u}^{\mu}} \text { and } \tilde{g}_{\mu \nu}=g_{\mu \nu}-\frac{k_{\mu} k_{\nu}}{k^{2}} .
$$

The transverse and longitudinal form factors have the property that, in the limit $\omega=0$ and $k \rightarrow 0, \Pi_{T}$ turns out to be zero and $\Pi_{L} \rightarrow \omega_{p}^{2}$ [4] 51. The limit $\omega=0$ and $k \rightarrow 0$, also termed "the" infrared limit, remains an interesting one to study the long wavelength paradigm of the gauge field excitations in a finite density medium. One can analyse infrared dynamics of the system in configuration space by first taking the limit mentioned before, followed by replacing $k^{i} \rightarrow i \partial^{i}$ in eqn. (2.6). The addition of the background medium induced pieces to the effective Lagrangian may not change the number of degrees of freedom of the system always. For instance, in presence of a background external electro-magnetic field, the number of physical degrees of freedom of quantum corrected U(1) gauge theory with fermions, remains the same as that of the free theory. However, the same may not be true when the medium induced quantum corrections are incorporated. The presence of unphysical degrees of freedom in a dynamical system can be inferred from the hessian matrix of the same. If the hessian matrix,

$$
\frac{\partial^{2} L_{e f f}}{\partial \dot{A_{\mu}} \partial \dot{A_{\nu}}}
$$

is noninvertible, the system is constrained, i.e., the number of dynamical variables are more than the number of physical degrees of freedom present in the system. In that case, to analyse the dynamics of the system one needs to follow the procedures out lined in [52 55]. With finite density effects, incorporated (in the effective Lagrangian) this method, may get very complicated due to presence of higher derivative terms. However, once the procedures (of constraints analysis) are completed, one can find the number of physical degrees of freedom $(N)$ from the equation,

$$
N=\frac{N_{p s v}-2 \times n_{1}-n_{2}}{2}
$$

where, $N_{p s v}$ stands for number of phase space variables, $n_{1}$ stands for the number of first class constraints, and $n_{2}$ stands for number of second class constraints.

One can, however infer the number of physical degrees of freedom for a system made up of material medium, with lesser effort, if one considers taking the infrared limit we discussed earlier. In this limit the full in medium effective Lagrangian $\left(L_{e f f(m)}\right)$ takes the form,

$$
L_{e f f(m)}=-\frac{1}{4} F_{\mu \nu} F^{\mu \nu}+\frac{1}{2} \omega_{p}^{2} A_{0} A_{0}
$$

barring the Lorentz structure, this Lagrangian is very close to the Lagrangian of the Proca model. This model although is gauge non-invariant, but known to produce correct number of degrees of freedom present in a massive theory. This system is known to have two second class constraints and no first class constraints. Therefore, the number of physical degrees of freedom, for the same, turns out to be three. Hence one needs to remove one of the four components of the gauge potential, of U(1) gauge theory, to describe the dynamics of the system. We expect the same to hold good in our case. The same is performed in the next paragraph. 


\subsection{Gauge fixing:}

In continuation to the discussion presented in the last paragraph, we consider $A_{g f}$, that appears in the equation for the gauge potential,

$$
A^{\nu}(k)=A_{\|}(k) \hat{b}^{(1) \nu}+A_{\perp}(k) \hat{I}^{\nu}+A_{L}(k) \hat{\tilde{u}}^{\nu}+A_{g f}(k) \hat{k}^{\nu},
$$

to be a redundant DOF and consider it to be equal to zero. As a consequence, it turns out that $k_{\nu} A^{\nu}(k)=0$ that happens to be the Lorentz gauge condition. We would like to point out at this stage that basis vectors $b^{(1) \nu}, I^{\nu}$ and $\tilde{u}^{\nu}$ used in equation (S64) to describe the gauge potential $A^{\nu}(k)$ can always be rotated to a set of new basis vectors, however the associated DOF in that basis may not be suitable for normal mode analysis of the system.

\subsection{Some Interesting observations:}

We further note here that equations (2.4) and (2.5) offer some interesting possibilities: in terms of them one can further define an effective metric in the momentum space as:

$$
G_{\mu \nu}=\frac{k_{\mu} k_{\nu}}{k^{2}}-\frac{b_{\mu}^{(1)} b_{\nu}^{(1)}}{b_{\alpha}^{(1)} b^{(1) \alpha}}-\frac{I_{\mu} I_{\nu}}{I_{\alpha} I^{\alpha}}-\frac{\tilde{u}_{\mu} \tilde{u}_{\nu}}{\tilde{u}_{\alpha} \tilde{u}^{\alpha}}
$$

using the orthogonal properties of the unit vectors it would be possible to to raise or lower the index of any general four-vector in momentum space using equation (2.12). The other important consequence that follows from the definition of the vector potential given by equation (S64) is that, the gauge fixed 4 -vector potential is space like irrespective of the choice of the momentum.

\subsection{Discrete symmetries}

The discrete symmetry $(\mathbf{C}, \mathbf{P}$ and $\mathbf{T})$ transformation properties of the tensors, four vectors and the EM form-factors, used here are listed in table [1. These transformation laws (agrees with ones provided in [56 59]) can be obtained using standard QFT based arguments, except for $u^{\mu}$ ( which can be obtained following the subtle principles of finite temperature field theory [20]).

\begin{tabular}{||c|ccccccccccccc||}
\hline & $F_{\mu \nu}$ & $k_{\mu}$ & $u_{\mu}$ & $\tilde{u}_{\mu}$ & $b_{\mu}^{(1)}$ & $b_{\mu}^{(2)}$ & $I_{\mu}$ & $A_{\|}$ & $A_{\perp}$ & $A_{L}$ & $f_{\mu \nu}$ & $i$ & $\epsilon_{\mu \nu \rho \sigma}$ \\
\hline \hline $\mathbf{C}$ & $-F_{\mu \nu}$ & $k_{\mu}$ & $-u_{\mu}$ & $-\tilde{u}_{\mu}$ & $-b_{\mu}^{(1)}$ & $-b_{\mu}^{(2)}$ & $-I_{\mu}$ & $A_{\|}$ & $A_{\perp}$ & $A_{L}$ & $-f_{\mu \nu}$ & $i$ & $\epsilon_{\mu \nu \rho \sigma}$ \\
$\mathbf{P}$ & $F^{\mu \nu}$ & $k^{\mu}$ & $u^{\mu}$ & $\tilde{u}^{\mu}$ & $b^{(1) \mu}$ & $b^{(2) \mu}$ & $I^{\mu}$ & $A_{\|}$ & $A_{\perp}$ & $A_{L}$ & $f_{\mu \nu}$ & $i$ & $-\epsilon_{\mu \nu \rho \sigma}$ \\
$\mathbf{T}$ & $-F^{\mu \nu}$ & $k^{\mu}$ & $-u^{\mu}$ & $-\tilde{u}^{\mu}$ & $-b^{(1) \mu}$ & $-b^{(2) \mu}$ & $-I^{\mu}$ & $-A_{\|}$ & $-A_{\perp}$ & $-A_{L}$ & $-f_{\mu \nu}$ & $-i$ & $-\epsilon_{\mu \nu \rho \sigma}$ \\
\hline \hline
\end{tabular}

Table 1: Transformation properties for the vectors, tensors and the EM form-factors used to describe $A^{\nu}(k)$ in equation (64), under $\mathbf{C}, \mathbf{P}$ and $\mathbf{T}$.

The question that one would like to pose next is, how the interaction between charge neutral spin zero scalar with charge neutral spin one photon, does take place. The best way to address the same is to take a discrete symmetry based route, done elegantly by Raffelt and Stodolosky in [15], invoking CP symmetry based arguments. Where in the case of a pseudo-scalar-photon (a $\gamma$ ) interacting system, it was shown that, in such situation, the $\mathbf{C P}$ asymmetric pseudo-scalar (axion) would couple to the $\mathbf{C P}$ asymmetric part of the photon's four vector potential. The CP symmetric part of the photon would remain decoupled. That is the $\mathbf{C P}$ violating helicity state (HS) of photon would couples with the $\mathbf{C P}$ violating axion field and evolve in space and time; and the $\mathbf{C P}$ preserving helicity state of the photon propagates freely. The dilaton photon dynamics, in similar situation, can be cast in a similar language 
as that of [15], exchanging the role of pseudo-scalars with scalars and scalars with pseudo-scalars.

Instead of following [15, we describe such a system here in terms of a set of EM form-factors of the photon, described by equation (S64), introduced originally in [60. The proof of these transformations properties are provided in the supplementary document of this article. Having these transformation laws in hand, we argue here, that, the equations of motion, when cast in terms of the electromagnetic form factors of table 1, follow a PT symmetry based coupling dynamics, instead of the $\mathbf{C P}$ symmetry based one of [15].

\section{Incorporation of matter effects}

Matter effects are incorporated through the inclusion of a term of the following form, $A^{\mu}(k) \Pi_{\mu \nu}(T, \mu) A^{\nu}(k)$ in the effective Lagrangian $\left(L_{e f f}\right)$ of the system 60 67. This is a scalar made up by contracting in-medium photon self-energy tensor $\Pi_{\mu \nu}(T, \mu)$ with gauge fields. Parameters $\mathrm{T}$ and $\mu$ stand for temperature and chemical potential as arguments of $\Pi_{\mu \nu}(T, \mu)$. The contribution of the parity violating part of weakly magnetized matter effects is similarly taken into account by the inclusion of a term like $A^{\mu}(k) \Pi_{\mu \nu}^{p}(k, \mu, T, e B) A^{\nu}(k)$, when $\Pi_{\mu \nu}^{p}(k, \mu, T, e B)$ is the photon polarization tensor evaluated by incorporating the effects of the magnetic field to first order in the external field strength $e B$ but exact to all orders in $\mathrm{T}$, and chemical potential $\mu$; using Schwinger's proper time propagator and formalism of finite temperature field theory [20,21,67. The photon polarization tensor $\Pi_{\mu \nu}^{p}(k)$ can be parameterised in the following way;

$$
\Pi_{\mu \nu}^{p}(k)=\Pi^{p}(k) P_{\mu \nu}, \text { when } \quad P_{\mu \nu}=i \epsilon_{\mu \nu \alpha \beta_{\|}} \frac{k^{\alpha}}{|k|} u^{\tilde{\beta}_{\|}},
$$

where $\epsilon_{\mu \nu \alpha \beta_{\|}}$is Levi-Civita tensor and subscript $\beta_{\|}=0,3$. In the expression for $P_{\mu \nu}, \tilde{\beta}_{\|}$is defined such that if $\beta_{\|}=0$ then $\tilde{\beta}_{\|}=3$ and vice-versa. We have discussed the $\mathbf{C}, \mathbf{P}$ and $\mathbf{T}$ symmetries of polarization tensors in the Supplementary materials part of this article.

\section{Equations of motion:}

Coupling between different DOF of the system, follows from the form of the effective Lagrangian. As can be verified from the equation (4.1) that, the couplings between $\mathbf{P T}$ violating $A_{\|}(k)$ and $\mathbf{P T}$ symmetric $\phi(k)$, is generated in the $\phi \gamma$ tree level Lagrangian (in external $B$ field), through a multiplicative $\mathbf{T}$ violating factor $i$. Thus making the Lagrangian $\mathbf{P T}$ symmetric. The other two PT violating EM form-factors $A_{\perp}(k)$ and $A_{L}(k)$ (see table 1) have no coupling with the scalar $\phi(k)$. Therefore, with the inclusion of $\Pi_{\mu \nu}(T, \mu)$, when $A_{L}(k)$ becomes non-zero, the mixing matrix for $\phi \gamma$ remains $2 \times 2$. But with the inclusion of the, effective Lagrangian due to parity violating magnetized-media-induced photon self-energy term $\Pi^{p}(k)_{\mu \nu}, A_{\|}(k)$ gets coupled to $A_{\perp}(k)$, thus turning the mixing matrix $3 \times 3$ one.

In magnetized media the effective Lagrangian- for $\phi \gamma$ interactive system including the effective interaction Lagrangians due to photon self-energy terms- is given by:

$$
L_{e f f, \phi}=\frac{1}{2} \phi\left[k^{2}-m_{\phi}^{2}\right] \phi-\frac{1}{4} f_{\mu \nu} f^{\mu \nu}+\frac{1}{2} A^{\mu} \Pi_{\mu \nu} A^{\nu}+\frac{1}{2} A^{\mu} \Pi_{\mu \nu}^{p} A^{\nu}-\frac{1}{4} g_{\phi \gamma \gamma} \phi \bar{F}^{\mu \nu} f_{\mu \nu} .
$$

In eqn. (4.1), the variable $f_{\mu \nu}$ stands for field strength for the dynamical photons, $\bar{F}_{\mu \nu}$ stands for the external field, $\Pi_{\mu \nu}$ photon polarization tensor in an isotropic medium, and $\Pi_{\mu \nu}^{p}$ the same in presence of magnetic field to $O(e B)$. 


\subsection{Mixing dynamics of $\phi \gamma$ interaction}

The equations of motion for the EM form-factors for the photon, in the notation of [60], turn out to be,

$$
\begin{aligned}
& \left(k^{2}-\Pi_{T}\right) A_{\|}(k)+i \Pi^{p}(k) N_{1} N_{2}\left[\epsilon_{\mu \nu \delta \beta} \frac{k^{\beta}}{|k|} u^{\tilde{\delta}_{\|}} b^{(1) \mu} I^{\nu}\right] A_{\perp}(k)=\frac{i g_{\phi \gamma \gamma} \phi(k)}{N_{1}}, \\
& \left(k^{2}-\Pi_{T}\right) A_{\perp}(k)-i \Pi^{p}(k) N_{1} N_{2}\left[\epsilon_{\mu \nu \delta \beta} \frac{k^{\beta}}{|k|} u^{\tilde{\delta}_{\|}} b^{(1) \mu} I^{\nu}\right] A_{\|}(k)=0, \\
& \left(k^{2}-\Pi_{L}\right) A_{L}(k)=0 .
\end{aligned}
$$

The three equations (i.e., (4.2)-(4.4) ), describe the dynamics of the three DOF of the photon. And the equation of motion for $\phi(k)$ is given by,

$$
\left(k^{2}-m^{2}\right) \phi(k)=-\frac{i g_{\phi \gamma \gamma} A_{\|}(k)}{N_{1}} .
$$

It can be checked that, left and right sides of the equations of motion above are $\mathbf{P T}$ symmetric. Once the discrete transformations $\mathbf{P}$ and $\mathbf{T}$ are applied on the variables on both side including background field $\left(\bar{F}^{\mu \nu}\right)$, EM form-factors of the photons and the scalar field $\phi(k)$, then it would show that equations of motion (4.2 to 4.5) remain invariant. For notational convenience, we next introduce the new variables $F$ and $G$, defined as,

$$
F=N_{1} N_{2} \Pi^{p}(k)\left[\epsilon_{\mu \nu \delta \beta} \frac{k^{\beta}}{|k|} u^{\tilde{\delta}_{\|}} b^{(1) \mu} I^{\nu}\right] \quad \text { and } \quad G=\frac{g_{\phi \gamma \gamma}}{N_{1}} .
$$

and would be using them when necessary.

As stated already, in the long wavelength limit, we consider $\Pi_{T}=\omega_{p}^{2}$, where $\omega_{p}=\sqrt{\frac{4 \pi \alpha n_{e}}{m_{e}}}$, is the plasma frequency, $\alpha$ is EM coupling constant and $n_{e}$ is the density of electrons. Other terms, $F$ and $G$ introduced in equation (4.6) can be simplified to yield $F=\frac{\omega_{p}^{2}}{\omega} \frac{e B \cos \theta}{m_{e}}$ and $G=-g_{\phi \gamma \gamma} B \sin \theta \omega$, where $\theta$ is angle between the photon propagation vector $\vec{k}$ and the magnetic field $B$. Here $m_{e}$ is the mass of electron and $e$ is the electronic charge. The equations of motion can now be cast in terms of a compact $4 \times 4$ matrix $\mathbf{M}^{\prime}$ as:

$$
\left[k^{2} \mathbf{I}-\mathbf{M}^{\prime}\right]\left(\begin{array}{c}
A_{\|}(k) \\
A_{\perp}(k) \\
A_{L}(k) \\
\phi(k)
\end{array}\right)=0
$$

As we have already mentioned that for $\phi \gamma$ system in magnetized media, the longitudinal DOF of photon does not mix with rest others, therefore we will exclude this term from the mixing matrix, and upon doing so, the $4 \times 4$ mixing matrix $\mathbf{M}^{\prime}$, reduces to a $3 \times 3$ mixing matrix denoted by $\mathbf{M}$. For the sake of brevity, using shorthand notations the equations of motions now can be written as follows:

$$
\left[k^{2} \mathbf{I}-\mathbf{M}\right]\left(\begin{array}{c}
A_{\|}(k) \\
A_{\perp}(k) \\
\phi(k)
\end{array}\right)=0
$$

where the $3 \times 3$ mixing matrix for scalar-photon interaction is given as;

$$
\mathbf{M}=\left(\begin{array}{ccc}
\omega_{p}^{2} & i F & -i G \\
-i F & \omega_{p}^{2} & 0 \\
i G & 0 & m_{\phi}^{2}
\end{array}\right)
$$

In order to get the Stoke parameters $\mathbf{I}(\omega, z), \mathbf{Q}(\omega, z), \mathbf{U}(\omega, z)$ and $\mathbf{V}(\omega, z)$ for the EM radiation, we need to get the solutions of the field equations; this can be achieved by diagonalizing $\mathbf{M}$. 


\subsection{Diagonalizing the $3 \times 3$ mixing matrix}

Our objective here is to obtain the analytical expression for the unitary matrix $\mathbf{U}$, that would diagonalize the hermitian matrix $\mathbf{M}$. We express the elements of the same $(\mathbf{U})$, using analytic algebraic expressions. Using this matrix we carry out the numerical operations, to estimates of observables, maintaining a numerical accuracy of the order $\sim 10^{-9}$ or more for the identities those the various intermediate expressions of interest need to satisfy during the numerical evaluation of the form factors.

In order to obtain the elements of the matrix $\mathbf{U}$, we need to solve the characteristic equation, obtained from $\operatorname{Det}\left(\mathbf{M}-\lambda_{j} \mathbf{I}\right)=0$, and find the eigen values (roots) i.e., $\lambda_{j}(j=1,2$ and 3$)$ of $\mathbf{M}$. Then use the same to find the corresponding eigen vectors. Finally, using the eigen vectors, construct the unitary matrix $\mathbf{U}$ that would diagonalize $\mathbf{M}$. The characteristic equation for this $3 \times 3$ hermitian matrix $\mathbf{M}$, for obvious reasons turns out to be a cubic equation, having real roots. The cubic equation, that follows from the characteristic equation can be written, in terms of parameters b, c and d as,

$$
\lambda_{j}^{3}+b \lambda_{j}^{2}+c \lambda_{j}+d=0
$$

where the parameters b, $\mathrm{c}$ and $\mathrm{d}$ are functions of the elements of mixing matrix $\mathbf{M}$, denoted by:

$$
\begin{aligned}
b & =-\left(2 \omega_{p}^{2}+m_{\phi}^{2}\right) \\
c & =\omega_{p}^{4}+2 \omega_{p}^{2} m_{\phi}^{2}-\left(\frac{e B_{\|}}{m_{e}} \frac{\omega_{p}^{2}}{\omega}\right)^{2}-\left(g_{\phi \gamma \gamma} B_{\perp} \omega\right)^{2} \\
d & =-\left[\omega_{p}^{4} m_{\phi}^{2}-\left(\frac{e B_{\|}}{m_{e}} \frac{\omega_{p}^{2}}{\omega}\right)^{2} m_{\phi}^{2}-\left(g_{\phi \gamma \gamma} B_{\perp} \omega\right)^{2} \omega_{p}^{2}\right] .
\end{aligned}
$$

Next we introduce the variables $P$ and $Q$, when $P=\left(\frac{3 c-b^{2}}{9}\right)$ and $2 Q=\left(\frac{2 b^{3}}{27}-\frac{b c}{3}+d\right)$; in terms of them, the roots turn out to be,

$$
\begin{aligned}
& \lambda_{1}=\mathbf{R} \cos \alpha+\sqrt{3} \mathbf{R} \sin \alpha-b / 3, \\
& \lambda_{2}=\mathbf{R} \cos \alpha-\sqrt{3} \mathbf{R} \sin \alpha-b / 3, \\
& \lambda_{3}=-2 \mathbf{R} \cos \alpha-b / 3,
\end{aligned} \quad \text { with } \quad\left\{\begin{array}{c}
\alpha=\frac{1}{3} \cos ^{-1}\left(\frac{Q}{\mathbf{R}^{3}}\right) \\
\mathbf{R}=\sqrt{(-P)} \operatorname{sgn}(Q) .
\end{array}\right.
$$

It should be noted that, in principle $\mathbf{R}$ can be equal to $+\sqrt{(-P)}$ or $-\sqrt{(-P)}$. However, the ratio $\left(\frac{Q}{\mathbf{R}^{3}}\right)$ should be positive. Hence to maintain the same, the factor of $\operatorname{sgn}(Q)$ is introduced in the definition of $\mathbf{R}$. The orthonormal eigenvectors $\mathbf{X}_{j}$ of $\mathbf{M}$ are to be found from the matrix relation, $\left[\mathbf{M}-\lambda_{j} \mathbf{I}\right]\left[\mathbf{X}_{j}\right]=0$. In terms of its elements, the normalized column vector $\left[\mathbf{X}_{j}\right]$, can be denoted as,

$$
\left[\mathbf{X}_{j}\right]=\left[\begin{array}{c}
\bar{u}_{j} \\
\bar{v}_{j} \\
\bar{w}_{j}
\end{array}\right]
$$

Following standard methods, one can evaluate these elements, in terms of the roots $\lambda_{j}$ and elements of the mixing matrix $\mathbf{M}$. The same, once evaluated turns out to be,

$$
\begin{aligned}
& \bar{u}_{j}=\left(\omega_{p}^{2}-\lambda_{j}\right)\left(m_{\phi}^{2}-\lambda_{j}\right) \times \mathcal{N}_{\mathrm{vn}}^{(\mathrm{j})}, \\
& \bar{v}_{j}=i \frac{e B_{\|}}{m_{e}} \frac{\omega_{p}^{2}}{\omega}\left(m_{\phi}^{2}-\lambda_{j}\right) \times \mathcal{N}_{\mathrm{vn}}^{(\mathrm{j})}, \\
& \bar{w}_{j}=i g_{\phi \gamma \gamma} B_{\perp} \omega\left(\omega_{p}^{2}-\lambda_{j}\right) \times \mathcal{N}_{\mathrm{vn}}^{(\mathrm{j})},
\end{aligned} \quad \text { when } \quad\left\{\mathcal{N}_{\mathrm{vn}}^{(\mathrm{j})}=\frac{1}{\sqrt{\left[\left|\overline{\mathrm{u}}_{\mathrm{j}}\right|^{2}+\left|\overline{\mathrm{v}}_{\mathrm{j}}\right|^{2}+\left|\overline{\mathrm{w}}_{\mathrm{j}}\right|^{2}\right]}}\right.
$$

Here $\mathcal{N}_{\mathrm{vn}}^{(\mathrm{j})}$ is normalisation constant and $j$ can take values from 1 to 3 . Using these eigenvectors, the unitary matrix $\mathbf{U}$ turns out to be : 


$$
\mathbf{U}=\left(\begin{array}{ccc}
\left(\omega_{p}^{2}-\lambda_{1}\right)\left(m_{\phi}^{2}-\lambda_{1}\right) \mathcal{N}_{\mathrm{vn}}^{(1)} & \left(\omega_{p}^{2}-\lambda_{2}\right)\left(m_{\phi}^{2}-\lambda_{2}\right) \mathcal{N}_{\mathrm{vn}}^{(2)} & \left(\omega_{p}^{2}-\lambda_{3}\right)\left(m_{\phi}^{2}-\lambda_{3}\right) \mathcal{N}_{\mathrm{vn}}^{(3)} \\
i \frac{e B_{\|}}{m_{e}} \frac{\omega_{p}^{2}}{\omega}\left(m_{\phi}^{2}-\lambda_{1}\right) \mathcal{N}_{\mathrm{vn}}^{(1)} & i \frac{e B_{\|}}{m_{e}} \frac{\omega_{p}^{2}}{\omega}\left(m_{\phi}^{2}-\lambda_{2}\right) \mathcal{N}_{\mathrm{vn}}^{(2)} & i \frac{e B_{\|}}{m_{e}} \frac{\omega_{p}^{2}}{\omega}\left(m_{\phi}^{2}-\lambda_{3}\right) \mathcal{N}_{\mathrm{vn}}^{(3)} \\
i g_{\phi \gamma \gamma} B_{\perp} \omega\left(\omega_{p}^{2}-\lambda_{1}\right) \mathcal{N}_{\mathrm{vn}}^{(1)} & i g_{\phi \gamma \gamma} B_{\perp} \omega\left(\omega_{p}^{2}-\lambda_{2}\right) \mathcal{N}_{\mathrm{vn}}^{(2)} & i g_{\phi \gamma \gamma} B_{\perp} \omega\left(\omega_{p}^{2}-\lambda_{3}\right) \mathcal{N}_{\mathrm{vn}}^{(3)}
\end{array}\right)
$$

The unitary matrix given by (4.16) is the one that diagonalizes the $3 \times 3$ mixing matrix $\mathbf{M}$.

\subsection{Field equation : Solutions}

In order to obtain the solutions of the coupled equation (4.8), one can multiply the same by inverse of the matrix given by eqn.(4.16) i.e., $\mathbf{U}^{-1}$ from left and use the property of unitary matrices $\mathbf{U U}^{-1}=\mathbf{1}$ in the same equation (i.e., (4.8), and arrive at,

$$
\mathbf{U}^{-1}\left[k^{2} \mathbf{I}-\mathbf{M}\right] \mathbf{U U}^{-1}\left(\begin{array}{c}
A_{\|}(k) \\
A_{\perp}(k) \\
\phi(k)
\end{array}\right)=\left[k^{2} \mathbf{I}-\mathbf{M}_{D}\right]\left(\begin{array}{c}
A_{\|}^{\prime}(k) \\
A_{\perp}^{\prime}(k) \\
\phi^{\prime}(k)
\end{array}\right)=0 .
$$

In order to arrive at equation (4.17), we have used the following notation,

$$
\left(\begin{array}{c}
A_{\|}^{\prime}(k) \\
A_{\perp}^{\prime}(k) \\
\phi^{\prime}(k)
\end{array}\right)=\mathbf{U}^{-1}\left(\begin{array}{c}
A_{\|}(k) \\
A_{\perp}(k) \\
\phi(k)
\end{array}\right) \text {. }
$$

The Matrix $\mathbf{M}_{D}$ is the diagonal matrix given by $\mathbf{M}_{D}=\mathbf{U}^{-1} \mathbf{M U}$ that has eigen values of the matrix $\mathbf{M}$ as diagonal elements. For a propagating beam of photons in the $z$ direction, $k_{3}$ can be re-transformed back to $z$ by taking inverse Fourier transform. Furthermore one can express $k^{2} \approx 2 \omega\left(\omega-i \partial_{z}\right)$, without much loss of generality, to use in the equations of motion. As a result of these (algebraic manipulations, equation (4.17) assumes the following form,

$$
\left[\left(\omega-i \partial_{z}\right) \mathbf{I}-\left[\begin{array}{ccc}
\frac{\lambda_{1}}{2 \omega} & 0 & 0 \\
0 & \frac{\lambda_{2}}{2 \omega} & 0 \\
0 & 0 & \frac{\lambda_{3}}{2 \omega}
\end{array}\right]\right]\left[\begin{array}{c}
A^{\prime}{ }_{\|}(z) \\
A_{\perp}^{\prime}(z) \\
\phi^{\prime}(z)
\end{array}\right]=0 .
$$

It is now easy to solve the matrix equation (4.19) by introducing the variables, $\Omega_{\|}=\left(\omega-\frac{\lambda_{1}}{2 \omega}\right), \Omega_{\perp}=$ $\left(\omega-\frac{\lambda_{2}}{2 \omega}\right)$ and $\Omega_{\phi}=\left(\omega-\frac{\lambda_{3}}{2 \omega}\right)$. Instead of going into details, we can now directly write down the solutions for the column vector $[\mathbf{A}(z), \phi(z)]^{T}$; in matrix form, they are given by:

$$
\left[\begin{array}{c}
A_{\|}(z) \\
A_{\perp}(z) \\
\phi(z)
\end{array}\right]=\mathbf{U}\left[\begin{array}{ccc}
e^{-i \Omega_{\|} z} & 0 & 0 \\
0 & e^{-i \Omega_{\perp} z} & 0 \\
0 & 0 & e^{-i \Omega_{\phi} z}
\end{array}\right] \mathbf{U}^{-1}\left[\begin{array}{c}
A_{\|}(0) \\
A_{\perp}(0) \\
\phi(0)
\end{array}\right]
$$

The magnitudes of the elements of column vector $[\mathbf{A}(0), \phi(0)]^{T}$ in equation (4.20), are subject to the boundary conditions appropriate for the physical situations assumed to be prevailing at the origin. Using the same initial conditions, one can write down the solution of equation (4.20) for $\mathrm{A}_{\|}(\omega, z)$, and it is:

$$
\begin{aligned}
A_{\|}(\omega, z) & =\left(e^{-i \Omega_{\|} z} \bar{u}_{1} \bar{u}_{1}^{*}+e^{-i \Omega_{\perp} z} \bar{u}_{2} \bar{u}_{2}^{*}+e^{-i \Omega_{\phi} z} \bar{u}_{3} \bar{u}_{3}^{*}\right) A_{\|}(\omega, 0) \\
& +\left(e^{-i \Omega_{\|} z} \bar{u}_{1} \bar{v}_{1}^{*}+e^{-i \Omega_{\perp} z} \bar{u}_{2} \bar{v}_{2}^{*}+e^{-i \Omega_{\phi} z} \bar{u}_{3} \bar{v}_{3}^{*}\right) A_{\perp}(\omega, 0) .
\end{aligned}
$$

Similarly the perpendicular component $A_{\perp}(\omega, z)$, turns out to be,

$$
\begin{aligned}
A_{\perp}(\omega, z) & =\left(e^{-i \Omega_{\|} z} \bar{v}_{1} \bar{u}_{1}^{*}+e^{-i \Omega_{\perp} z} \bar{v}_{2} \bar{u}_{2}^{*}+e^{-i \Omega_{\phi} z} \bar{v}_{3} \bar{u}_{3}^{*}\right) A_{\|}(\omega, 0) \\
& +\left(e^{-i \Omega_{\|} z} \bar{v}_{1} \bar{v}_{1}^{*}+e^{-i \Omega_{\perp} z} \bar{v}_{2} \bar{v}_{2}^{*}+e^{-i \Omega_{\phi} z} \bar{v}_{3} \bar{v}_{3}^{*}\right) A_{\perp}(\omega, 0) .
\end{aligned}
$$

Here, the parameters $A_{\perp}(\omega, 0)$ and $A_{\|}(\omega, 0)$ present in equation (4.21) and (4.22) are $A_{\perp}(\omega, z)$ and $A_{\|}(\omega, z)$ respectively under the boundary conditions as mentioned above. 


\section{$5 \quad$ Polarimetric observables}

From the coherency matrix, Stokes parameters, can be obtained. In terms of the solutions of the field equations they can be expressed as:

$$
\begin{aligned}
\mathbf{I}(\omega, z) & =<A_{\|}{ }^{*}(\omega, z) A_{\|}(\omega, z)>+<A_{\perp}^{*}(\omega, z) A_{\perp}(\omega, z)>, \\
\mathbf{Q}(\omega, z) & =<A_{\|}{ }^{*}(\omega, z) A_{\|}(\omega, z)>-<A_{\perp}^{*}(\omega, z) A_{\perp}(\omega, z)>, \\
\mathbf{U}(\omega, z) & =2 \operatorname{Re}<A_{\|}{ }^{*}(\omega, z) A_{\perp}(\omega, z)>, \\
\mathbf{V}(\omega, z) & =2 \operatorname{Im}<A_{\|}^{*}(\omega, z) A_{\perp}(\omega, z)>.
\end{aligned}
$$

It should be noted that $\mathbf{V}(\omega, z)$ in the equation (5.1) is a measure of circular polarization. Other polarimetric observables, i.e., degree of linear polarization, ellipticity angle, polarization angle, follows from the expressions of $\mathbf{I}(\omega, z), \mathbf{Q}(\omega, z), \mathbf{U}(\omega, z)$ and $\mathbf{V}(\omega, z)$. The degree of linear polarization (represented as $P_{L}$ ) along with $\Pi$, are given by,

$$
\begin{aligned}
P_{L} & =\frac{\sqrt{\mathbf{Q}^{2}(\omega, z)+\mathbf{U}^{2}(\omega, z)}}{\mathbf{I}(\omega, z)}, \\
\Pi & =\frac{\mathbf{Q}(\omega, z)}{\mathbf{I}(\omega, z)}
\end{aligned}
$$

The polarization angle (represented by $\Psi_{p}$ ), is defined in terms of $\mathbf{U}$ and $\mathbf{Q}$, as:,

$$
\tan \left(2 \Psi_{p}\right)=\frac{\mathbf{U}(\omega, z)}{\mathbf{Q}(\omega, z)} .
$$

The ellipticity angle (denoted by $\chi$ ), is defined as:

$$
\tan (2 \chi)=\frac{\mathbf{V}(\omega, z)}{\sqrt{\mathbf{Q}^{2}(\omega, z)+\mathbf{U}^{2}(\omega, z)}} .
$$

\subsection{Stokes $\mathbf{I}(\omega, z)$ and $\mathbf{Q}(\omega, z)$}

To find out the expressions of Stokes parameters $\mathbf{I}(\omega, \mathrm{z})$ and $\mathbf{Q}(\omega, \mathrm{z})$ for Scalar-photon mixing, we need to evaluate $\left|A_{\|}(\omega, z)\right|^{2}$ and $\left|A_{\perp}(\omega, z)\right|^{2}$, using equations (4.21) and (4.22). Introducing the new variables, $\mathbb{P}=\left|\bar{u}_{1}\right|\left|\bar{v}_{1}\right|, \mathbb{Q}=\left|\bar{u}_{2}\right|\left|\bar{v}_{2}\right|$ and $\mathbb{R}=\left|\bar{u}_{3}\right|\left|\bar{v}_{3}\right|$, the expression for $\left|A_{\|}(\omega, z)\right|^{2}$ in terms of them, turns out to be,

$$
\begin{aligned}
\left|A_{\|}(\omega, z)\right|^{2}=[ & -4\left|\bar{u}_{1}\right|^{2}\left|\bar{u}_{2}\right|^{2} \sin ^{2}\left(\frac{\left(\Omega_{\|}-\Omega_{\perp}\right) z}{2}\right)-4\left|\bar{u}_{2}\right|^{2}\left|\bar{u}_{3}\right|^{2} \sin ^{2}\left(\frac{\left(\Omega_{\perp}-\Omega_{\phi}\right) z}{2}\right) \\
& \left.-4\left|\bar{u}_{3}\right|^{2}\left|\bar{u}_{1}\right|^{2} \sin ^{2}\left(\frac{\left(\Omega_{\phi}-\Omega_{\|}\right) z}{2}\right)\right] \times\left|A_{\|}(\omega, 0)\right|^{2} \\
& -4\left[\mathbb{P Q} \sin ^{2}\left(\frac{\left(\Omega_{\|}-\Omega_{\perp}\right) z}{2}\right)+\mathbb{Q R} \sin ^{2}\left(\frac{\left(\Omega_{\perp}-\Omega_{\phi}\right) z}{2}\right)\right. \\
& \left.+\mathbb{R} \mathbb{P} \sin ^{2}\left(\frac{\left(\Omega_{\phi}-\Omega_{\|}\right) z}{2}\right)\right] \times\left|A_{\perp}(\omega, 0)\right|^{2} \\
& +\left[\left|\bar{u}_{1} \bar{u}_{2}\right|\left(\left|\bar{u}_{1} \bar{v}_{2}\right|-\left|\bar{u}_{2} \bar{v}_{1}\right|\right) \sin \left(\left(\Omega_{\|}-\Omega_{\perp}\right) z\right)\right. \\
& +\left|\bar{u}_{2} \bar{u}_{3}\right|\left(\left|\bar{u}_{2} \bar{v}_{3}\right|-\left|\bar{u}_{3} \bar{v}_{2}\right|\right) \sin \left(\left(\Omega_{\perp}-\Omega_{\phi}\right) z\right) \\
& \left.+\left|\bar{u}_{3} \bar{u}_{1}\right|\left(\left|\bar{u}_{3} \bar{v}_{1}\right|-\left|\bar{u}_{1} \bar{v}_{3}\right|\right) \sin \left(\left(\Omega_{\phi}-\Omega_{\|}\right) z\right)\right] \\
& \times 2\left|A_{\|}(\omega, 0)\right|\left|A_{\perp}(\omega, 0)\right| .
\end{aligned}
$$


Similarly, we can find $\left|A_{\perp}(\omega, z)\right|^{2}$. The expression for the same, in terms of the variables introduced earlier, turns out to be,

$$
\begin{aligned}
\left|A_{\perp}(\omega, z)\right|^{2}=- & 4\left[\mathbb{P} \mathbb{Q} \sin ^{2}\left(\frac{\left(\Omega_{\|}-\Omega_{\perp}\right) z}{2}\right)+\mathbb{Q R} \sin ^{2}\left(\frac{\left(\Omega_{\perp}-\Omega_{\phi}\right) z}{2}\right)\right. \\
& \left.+\mathbb{R} \mathbb{P} \sin ^{2}\left(\frac{\left(\Omega_{\phi}-\Omega_{\|}\right) z}{2}\right)\right] \times\left|A_{\|}(\omega, 0)\right|^{2} \\
+ & {\left[1-4\left|\bar{v}_{1}\right|^{2}\left|\bar{v}_{2}\right|^{2} \sin ^{2}\left(\frac{\left(\Omega_{\|}-\Omega_{\perp}\right) z}{2}\right)-4\left|\bar{v}_{2}\right|^{2}\left|\bar{v}_{3}\right|^{2} \sin ^{2}\left(\frac{\left(\Omega_{\perp}-\Omega_{\phi}\right) z}{2}\right)\right.} \\
& \left.-4\left|\bar{v}_{3}\right|^{2}\left|\bar{v}_{1}\right|^{2} \sin ^{2}\left(\frac{\left(\Omega_{\phi}-\Omega_{\|}\right) z}{2}\right)\right] \times\left|A_{\perp}(\omega, 0)\right|^{2} \\
+ & {\left[\left|\bar{v}_{1} \bar{v}_{2}\right|\left(\left|\bar{u}_{1} \bar{v}_{2}\right|-\left|\bar{u}_{2} \bar{v}_{1}\right|\right) \sin \left(\left(\Omega_{\|}-\Omega_{\perp}\right) z\right)\right.} \\
& +\left|\bar{v}_{2} \bar{v}_{3}\right|\left(\left|\bar{u}_{2} \bar{v}_{3}\right|-\left|\bar{u}_{3} \bar{v}_{2}\right|\right) \sin \left(\left(\Omega_{\perp}-\Omega_{\phi}\right) z\right) \\
& \left.+\left|\bar{v}_{3} \bar{v}_{1}\right|\left(\left|\bar{u}_{3} \bar{v}_{1}\right|-\left|\bar{u}_{1} \bar{v}_{3}\right|\right) \sin \left(\left(\Omega_{\phi}-\Omega_{\|}\right) z\right)\right] \\
& \times 2\left|A_{\|}(\omega, 0)\right|\left|A_{\perp}(\omega, 0)\right| .
\end{aligned}
$$

Using equations (5.6) and (5.7), one can get the expressions for $\mathbf{I}(\omega, z)$ and $\mathbf{Q}(\omega, z)$ as follows:

$$
\begin{aligned}
\mathbf{I}(\omega, z) & =\left|A_{\|}(\omega, z)\right|^{2}+\left|A_{\perp}(\omega, z)\right|^{2} \\
\mathbf{Q}(\omega, z) & =\left|A_{\|}(\omega, z)\right|^{2}-\left|A_{\perp}(\omega, z)\right|^{2} .
\end{aligned}
$$

\subsection{Stokes $\mathrm{U}(\omega, z)$ and $\mathbf{V}(\omega, z)$}

The expressions for $\mathbf{U}(\omega, z)$ and $\mathbf{V}(\omega, z)$ can be written in terms of $\mathcal{U}_{\|}, \mathcal{U}_{\perp}, \mathcal{U}_{\| \perp}$ and $\mathcal{V}_{\|}, \mathcal{V}_{\perp}, \mathcal{V}_{\| \perp}$. For $\mathbf{U}(\omega, z)$ they are given by :

$$
\begin{aligned}
\mathcal{U}_{\|} & =\left|\bar{u}_{1} \bar{u}_{2}\right|\left(\left|\bar{v}_{1} \bar{u}_{2}\right|-\left|\bar{u}_{1} \bar{v}_{2}\right|\right) \sin \left(\left(\Omega_{\|}-\Omega_{\perp}\right) z\right)+\left|\bar{u}_{2} \bar{u}_{3}\right|\left(\left|\bar{v}_{2} \bar{u}_{3}\right|-\left|\bar{u}_{2} \bar{v}_{3}\right|\right) \sin \left(\left(\Omega_{\perp}-\Omega_{\phi}\right) z\right) \\
& +\left|\bar{u}_{3} \bar{u}_{1}\right|\left(\left|\bar{v}_{3} \bar{u}_{1}\right|-\left|\bar{u}_{3} \bar{v}_{1}\right|\right) \sin \left(\left(\Omega_{\phi}-\Omega_{\|}\right) z\right) . \\
& \mathcal{U}_{\perp}=\left|\bar{v}_{1} \bar{v}_{2}\right|\left(\left|\bar{v}_{1} \bar{u}_{2}\right|-\left|\bar{u}_{1} \bar{v}_{2}\right|\right) \sin \left(\left(\Omega_{\|}-\Omega_{\perp}\right) z\right)+\left|\bar{v}_{2} \bar{v}_{3}\right|\left(\left|\bar{v}_{2} \bar{u}_{3}\right|-\left|\bar{u}_{2} \bar{v}_{3}\right|\right) \sin \left(\left(\Omega_{\perp}-\Omega_{\phi}\right) z\right) \\
& +\left|\bar{v}_{3} \bar{v}_{1}\right|\left(\left|\bar{v}_{3} \bar{u}_{1}\right|-\left|\bar{u}_{3} \bar{v}_{1}\right|\right) \sin \left(\left(\Omega_{\phi}-\Omega_{\|}\right) z\right) . \\
& \mathcal{U}_{\| \perp}=\left(\left|\bar{v}_{1} \bar{u}_{2}\right|-\left|\bar{u}_{1} \bar{v}_{2}\right|\right)^{2} \cos \left(\left(\Omega_{\|}-\Omega_{\perp}\right) z\right)+\left(\left|\bar{v}_{2} \bar{u}_{3}\right|-\left|\bar{u}_{2} \bar{v}_{3}\right|\right)^{2} \cos \left(\left(\Omega_{\perp}-\Omega_{\phi}\right) z\right) \\
& +\left(\left|\bar{v}_{3} \bar{u}_{1}\right|-\left|\bar{u}_{3} \bar{v}_{1}\right|\right)^{2} \cos \left(\left(\Omega_{\phi}-\Omega_{\|}\right) z\right) .
\end{aligned}
$$

And similarly, the expressions for $\mathcal{V}_{\|}, \mathcal{V}_{\perp}$ and $\mathcal{V}_{\| \perp}$, introduced to express the measure of circular polarization $\mathbf{V}(\omega, z)$ are:

$$
\begin{aligned}
\mathcal{V}_{\|} & =\left[\left(\left|\bar{u}_{1} \bar{v}_{1}\right|\left|\bar{u}_{1}\right|^{2}+\left|\bar{u}_{2} \bar{v}_{2}\right|\left|\bar{u}_{2}\right|^{2}+\left|\bar{u}_{3} \bar{v}_{3}\right|\left|\bar{u}_{3}\right|^{2}\right)+\left|\bar{u}_{1} \bar{u}_{2}\right|\left(\left|\bar{v}_{1} \bar{u}_{2}\right|+\left|\bar{u}_{1} \bar{v}_{2}\right|\right) \cos \left(\left(\Omega_{\|}-\Omega_{\perp}\right) z\right)\right. \\
& \left.+\left|\bar{u}_{2} \bar{u}_{3}\right|\left(\left|\bar{v}_{2} \bar{u}_{3}\right|+\left|\bar{u}_{2} \bar{v}_{3}\right|\right) \cos \left(\left(\Omega_{\perp}-\Omega_{\phi}\right) z\right)+\left|\bar{u}_{3} \bar{u}_{1}\right|\left(\left|\bar{v}_{3} \bar{u}_{1}\right|+\left|\bar{u}_{3} \bar{v}_{1}\right|\right) \cos \left(\left(\Omega_{\phi}-\Omega_{\|}\right) z\right)\right] . \\
& \\
\mathcal{V}_{\perp} & =\left[\left(\left|\bar{u}_{1} \bar{v}_{1}\right|\left|\bar{v}_{1}\right|^{2}+\left|\bar{u}_{2} \bar{v}_{2}\right|\left|\bar{v}_{2}\right|^{2}+\left|\bar{u}_{3} \bar{v}_{3}\right|\left|\bar{v}_{3}\right|^{2}\right)+\left|\bar{v}_{1} \bar{v}_{2}\right|\left(\left|\bar{u}_{1} \bar{v}_{2}\right|+\left|\bar{v}_{1} \bar{u}_{2}\right|\right) \cos \left(\left(\Omega_{\|}-\Omega_{\perp}\right) z\right)\right. \\
& \left.+\bar{v}_{2} \bar{v}_{3}\left|\left(\left|\bar{u}_{2} \bar{v}_{3}\right|+\left|\bar{u}_{3} \bar{v}_{2}\right|\right) \cos \left(\left(\Omega_{\perp}-\Omega_{\phi}\right) z\right)+\right| \bar{v}_{3} \bar{v}_{1} \mid\left(\left|\bar{u}_{3} \bar{v}_{1}\right|+\left|\bar{u}_{1} \bar{v}_{3}\right|\right) \cos \left(\left(\Omega_{\phi}-\Omega_{\|}\right) z\right)\right] .
\end{aligned}
$$




$$
\begin{aligned}
\mathcal{V}_{\| \perp} & =\left[\left(\left|\bar{u}_{1}\right|^{2}\left|\bar{v}_{2}\right|^{2}-\left|\bar{u}_{2}\right|^{2}\left|\bar{v}_{1}\right|^{2}\right) \sin \left(\left(\Omega_{\|}-\Omega_{\perp}\right) z\right)+\left(\left|\bar{u}_{2}\right|^{2}\left|\bar{v}_{3}\right|^{2}-\left|\bar{u}_{3}\right|^{2}\left|\bar{v}_{2}\right|^{2}\right) \sin \left(\left(\Omega_{\perp}-\Omega_{\phi}\right) z\right)\right. \\
& \left.+\left(\left|\bar{u}_{3}\right|^{2}\left|\bar{v}_{1}\right|^{2}-\left|\bar{u}_{1}\right|^{2}\left|\bar{v}_{3}\right|^{2}\right) \sin \left(\left(\Omega_{\phi}-\Omega_{\|}\right) z\right)\right] .
\end{aligned}
$$

Using them, one can write the expressions for $\mathbf{U}(\omega, \mathrm{z})$ and $\mathbf{V}(\omega, \mathrm{z})$ as follows:

$$
\begin{aligned}
& \mathbf{U}(\omega, z)=\mathcal{U}_{\|} \times 2\left|A_{\|}(\omega, 0)\right|^{2}+\mathcal{U}_{\perp} \times 2\left|A_{\perp}(\omega, 0)\right|^{2}+\mathcal{U}_{\| \perp} \times 2\left|A_{\perp}(\omega, 0)\right|\left|A_{\|}(\omega, 0)\right| \\
& \mathbf{V}(\omega, z)=\mathcal{V}_{\|} \times 2\left|A_{\|}(\omega, 0)\right|^{2}+\mathcal{V}_{\perp} \times 2\left|A_{\perp}(\omega, 0)\right|^{2}+\mathcal{V}_{\| \perp} \times 2\left|A_{\perp}(\omega, 0)\right|\left|A_{\|}(\omega, 0)\right|
\end{aligned}
$$

The measures of linear and circular polarization $\mathbf{Q}(\omega, \mathrm{z}), \mathbf{U}(\omega, \mathrm{z})$ and polarization angle $\Psi_{p}$ are plotted as a function of energy in figures [1. The discussion about them is provided below.

\section{Possible Physical Situation}

Gamma ray bursts (GRB) are stellar sized explosions ( those usually found with an associated luminosity of about $\sim 10^{51} \mathrm{ergs} / \mathrm{sec}$ ), observed to occur isotropically at a red-shifts $z=1$ or $z=2$ with the EM radiation in the form of X-ray or gamma-ray, with fluence of the order of $10^{-6} \mathrm{ergs} / \mathrm{cm}^{2}$, as observed from earth [68. Their size is usually estimated from the time scale variability of their light curve, estimated to be around $0.1 \mathrm{sec}[69$.

Accordingly their size is estimated to be $\sim 10^{9} \mathrm{~cm}$. The energy is believed to be injected from an object, like neutron star, of radius $10^{6} \mathrm{~cm}$. The high the degree of linear polarization associated with the EM spectrum ( e.g. $\Pi=27 \pm 11 \%$ in GRBICO826A ), associated with them, makes one infer, a strong magnetic field $\sim 10^{9}$ Gauss to be associated with them. The plasma frequency $\omega_{p}$ associated with them are believed to lie above, $\omega_{p}>10^{-17} \mathrm{GeV}$ [70 72]. The mechanism behind this explosion is currently under scrutiny; one believes that the polarization studies with intensity of the spectra holds the key to understand the geometry of the magnetic field at the source and the energy release mechanism. Though these aspects can be studied using classical physics, however one needs to be cautious, since factors like, presence of ALPs also potentially contribute to the modification of these observables. Therefore any anomaly in these signature may provide a clue to the existence of ALPs.

\section{$7 \quad$ Results}

Assuming a global magnetic field of magnitude $\sim 10^{9}$ Gauss, to be existing in a GRB fireball- over a path length of $\sim 10^{9} \mathrm{~cm}$, the stokes parameters $\mathbf{Q}, \mathbf{U}$ and the polarization angle $\Psi_{p}$ were estimated using equation (5.1) and (5.4) numerically - for scalar-photon coupling constant $g_{\phi \gamma \gamma} \sim 10^{-11} \mathrm{GeV}^{-1}$ with $\omega_{p} \sim 10^{-13} \mathrm{GeV}$, and $m_{\phi} \sim 10^{-2} \mathrm{eV}$. The plots of the same are provided in figure [1.

Since the degree of linear polarization $\Pi$, turns out to be equal to $\mathbf{Q}$, when $\mathbf{U}$ is neglected (as is evident from the respective definitions of the same), we can get the information about the amplitude variation for $\mathbf{Q}, \boldsymbol{\Pi}$ and $\boldsymbol{\Psi}_{p}$ over the energy $(\omega)$ interval $1.0 \times 10^{-6} \mathrm{GeV}<\omega<1.0 \times 10^{-5} \mathrm{GeV}$ from the plots provided in figure [1.

In most of the astrophysically viable, satellite based experiments, the detectors are hardly line sensitive, they usually operate over a broad energy range; hence the parameters (of Stokes) are usually estimated by adding the signal strengths over an energy range (according to the detector under consideration), followed by an averaging of the signal - over that same energy range.

Now if we look into these plots, we notice the existence of the highly oscillating part for the estimates of $\mathbf{Q}$ in the energy interval, $1.0 \times 10^{-6} \mathrm{GeV}<\omega<3.0 \times 10^{-6} \mathrm{GeV}$ and a similar pattern also for $\mathbf{U}$ in the interval $1.0 \times 10^{-6} \mathrm{GeV}<\omega<2.0 \times 10^{-6} \mathrm{GeV}$. This is followed by an monotonically increasing or decreasing pattern (left panel of figure [1). Similar effect, is identifiable in the plot for $\Psi_{p}$ vs energy in the right panel of the same figure (i.e., figure 1). 

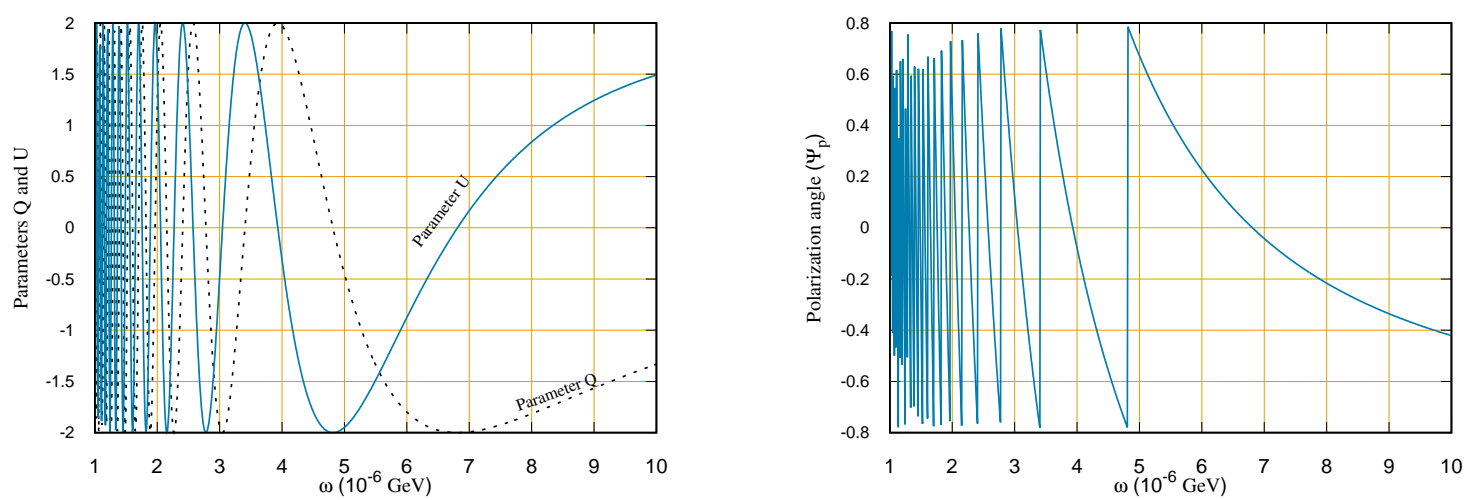

Figure 1: (In left), plot of Stokes parameter $\mathbf{Q}$ and $\mathbf{U}$ vs energy $(\omega)$. (In right), plot of polarization angle $\left(\Psi_{p}\right)$ vs energy $(\omega)$. Here, $m_{\phi}=1.0 \times 10^{-12} \mathrm{GeV}$ and $\left.\omega_{p}=3.7 \times 10^{-13} \mathrm{GeV}\right)$.

Therefore if the standard satellite based experiment dictated data extraction prescriptions are followed - while extracting signals from the datum like that producing figure 1-it would lead to the generation of an extremely low strength unphysical signal. This happens due to a major cancellation of the contributions due to strong oscillations of the actual signals around zero.

Thus, in order to get a realistic and statistically significant estimate of a signal, from detectors operating over a broad energy range, one must explore the available parameter space, over which the variations of the signals are stable with energy.

A collection of data sets for the spectro-polarimetric observables, those (unlike the ones of figure 11) looked stable under the variation with respect to $\omega$ ), were found in the $1-10 \mathrm{KeV}$ range, when the numerical size of the parameters $\omega_{p}$, and $m_{\phi}$ were considered at around $\sim 10^{-15} \mathrm{GeV}$, while the path length $(z)$, magnetic field $\operatorname{strength}(e B)$ and scalar photon coupling strength $\left(g_{\phi \gamma \gamma}\right)$ were maintained at $10^{6} \mathrm{~cm}, e B \sim 10^{9}$ Gauss and, $g_{\phi \gamma \gamma} \sim 10^{-11} \mathrm{GeV}^{-1}$, respectively.

The plots of the spectro-polarimetric observables, such as linear polarization $\left(P_{L}\right)$, polarization angle $\Psi_{p}$, ellipticity angle $\chi$ and degree of polarization $\Pi$, estimated with these parameters, are displayed in figure 2, for an energy interval- in the 1-10 KeV range. Interestingly enough, the estimates of maximum linear polarization, for these parameters and energy range, is about $99 \%$ and the polarization angle is about $12^{\circ}$; those lie well within the range of the observed linear-polarization and polarization angle estimated from satellite borne astrophysical observations, for the GRBs, occurring across the sky.

This little interesting exercise could have been used to project out a possible operating energy range for the space borne detectors, to explore the existence of ALPs in the parameter range considered above; had it not have faced a constraint (veto) from the fifth-force experiments due to dilatons, that we elaborate below.

\subsection{Fifth force constraints}

The scale symmetry, thats being investigated in this work, is an approximate one. This is due to the presence of massive dilatons. In field theory context, it can happen through the incorporation of higher dimensional conformal symmetry breaking operators (having scaling dimension other than four ) or by breaking the symmetry spontaneously as was done in 6 .

And in unified theories of extra dimension, this breaking may be due to nonzero curvature associated with the extra dimension. Whatever may be the origin, their existence would modify, the predictions of gravitational force of Einstein gravity with dilatonic fifth force. Experiments performed with torsionbalance, to find the range of dilatonic fifth force, puts an upper bound on dilaton mass to be $\sim O\left(10^{-11}\right)$ 

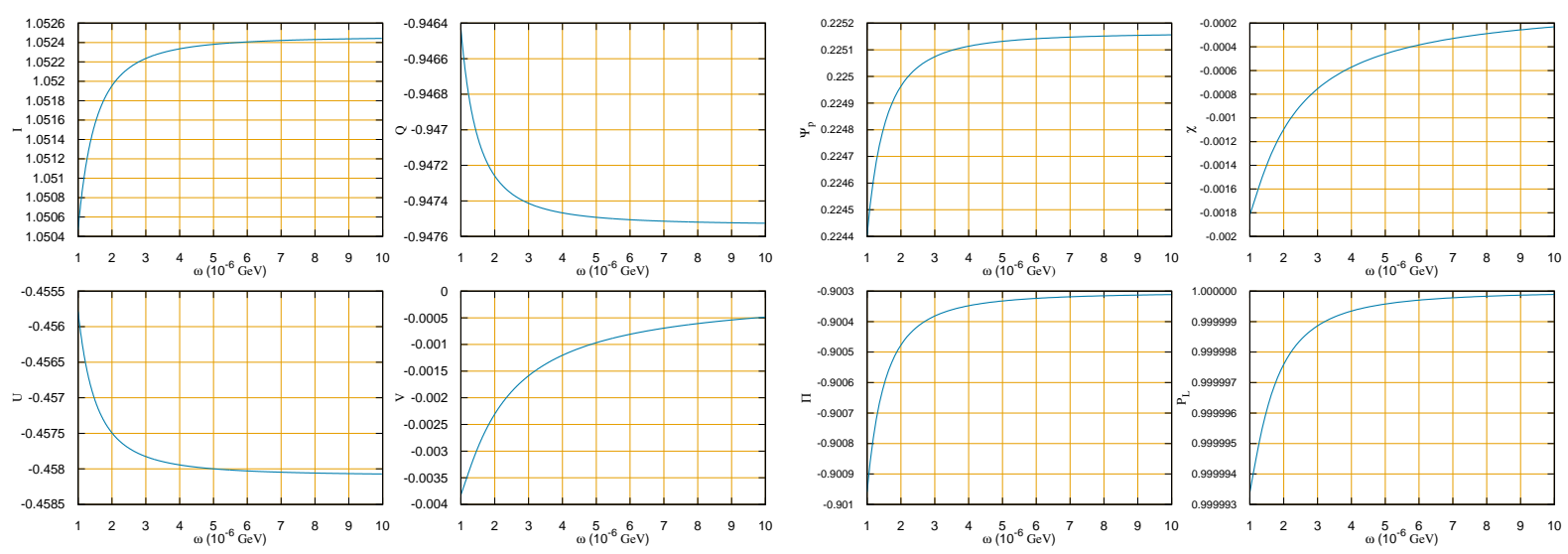

Figure 2: (In left panel) plots of Stokes parameters (I, Q, U , V) vs energy $(\omega)$. (In right panel) plots of polarization angle ( $\Psi_{p}$, ellipticity angle $(\chi)$, degree of polarization $(\Pi)$ and linear polarization $\left(P_{L}\right)$ vs energy $(\omega)$. Here, $m_{\phi}=1.0 \times 10^{-15} \mathrm{GeV}$ and $\omega_{p}=3.7 \times 10^{-15} \mathrm{GeV}$.
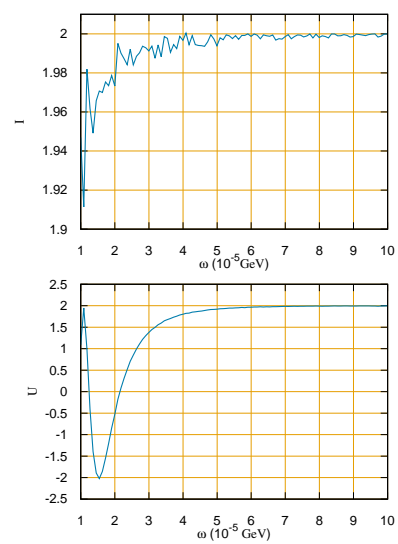
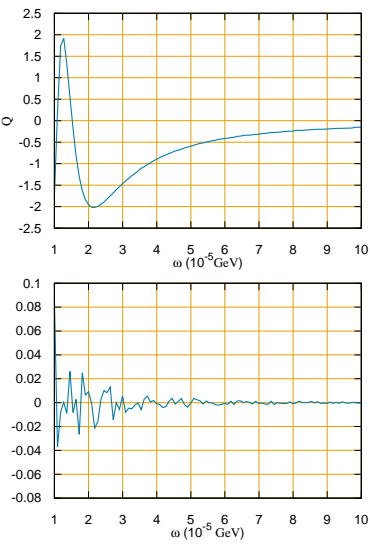
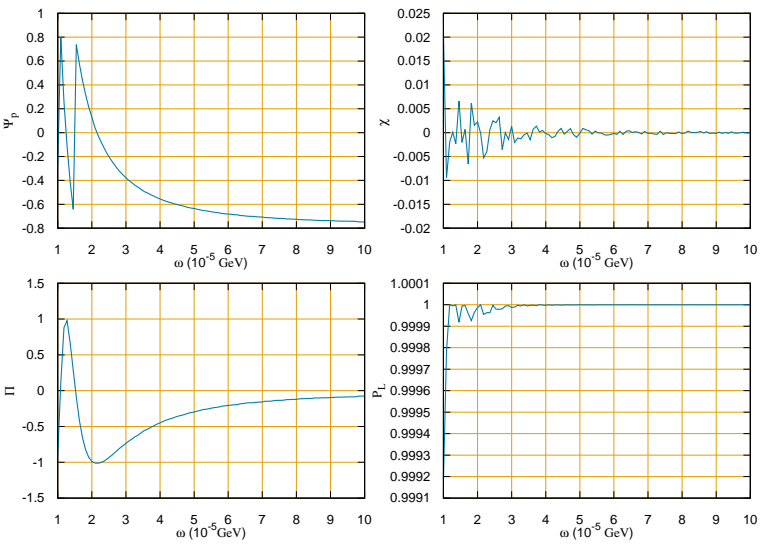

Figure 3: (In left panel) plots of Stokes parameters $(\mathbf{I}, \mathbf{Q}, \mathbf{U}, \mathbf{V})$ vs energy $(\omega)$. (In right panel) plots of polarization angle ( $\Psi_{p}$, ellipticity angle $(\chi)$, degree of polarization $(\Pi)$ and linear polarization $\left(P_{L}\right)$ vs energy $(\omega)$. Parameters considered here i.e., $m_{\phi}=1.0 \times 10^{-11} \mathrm{GeV}$ and $\omega_{p}=3.7 \times 10^{-10} \mathrm{GeV}$, respecting fifth force constraints.

$\mathrm{GeV}$. Hence, one needs to consider the value of $m_{\phi} \sim 10^{-11} \mathrm{GeV}$, for any realistic search for $\phi$.

Although we had seen earlier, that, the change in the spectro-polarimetric observables with energy $(\omega)$ were marginal when the mass of $m_{\phi}$ and plasma frequency $\omega_{p}$ were of the order of $\sim 10^{-15} \mathrm{GeV}$; but due to the reasons explained above those results are unrealistic.

In the light of this, (i.e., for $m_{\phi} \geq 10^{-2} \mathrm{eV}$ ), identifying the favourable regions in the parameter variables (i.e., estimates of $\omega_{p} e B$ and $g_{\phi \gamma \gamma}$ etc.) over which the signals would remain stabilised-is a time consuming task. A way of bypassing this apparent difficulty, is by scaling these variables with $m_{\phi}$ and finding the magnitude of the changed variables, such that, the scaled ones remain constant. These new modified values are expected to provide a suitable parameter space, that one is searching for. We had performed this scaling exercise. And found the following modified values for the variables $\omega_{p}=3.7 \times 10^{-10}$ $\mathrm{GeV}, e B=1.95 \times 10^{-16} \mathrm{GeV}^{2}$ and $g_{\phi \gamma \gamma}=1.0 \times 10^{-14} \mathrm{GeV}$, rest being the same. The results are provided in figure 3. The numerical exercise confirms the existence of of stable signals in the energy range $1.0 \times 10^{-5} \mathrm{GeV}<\omega<1.0 \times 10^{-4} \mathrm{GeV}$, for the scaled variables, chosen here. 
However, in the process, the original parameters, chosen from the generally accepted models of Gamma Ray Bursts, got modified. So one is left with the option of exploring refined models of GRB emission, like synchrotron emission based models or hydrodynamic instability based models or streaming instability based models etc., as described in [ 73]- [81] ].

\section{Discussion}

For obvious reasons, detecting X-ray or gamma- ray signals from earth surface is difficult. Hence space borne detectors(e.g., GAP) have been developed and used to detect the same [71. They happen to be sensitive to a wide energy range; for instance GAP is sensitive to an energy range $70-300 \mathrm{KeV}$. The Ranaty High Energy Solar Spectrometer Imager (RHESSI) [74] had an operational window of 0.15 -2 $\mathrm{MeV}$, the same for SWIFT satellite 75, happen to be $300 \mathrm{KeV}-10 \mathrm{MeV}$ etc. On the other hand our numerical estimates show that, the signals may undergo huge amount of oscillations in these operational windows. A crucial assumption that goes in the detection of polarization signal is additivity of the Stokes Parameters over an energy range. And the difference in the polarization angles of the GRB EM beam, at two ends of the band, to be less than ninety degree, i.e., 72

$$
\left|\Delta\left(E_{2}, z\right)-\Delta\left(E_{1}, z\right)\right| \leq \frac{\pi}{2} .
$$

This may not always be the case, as our numerical estimates show. Since many of the polarization dataGRB or others- are used to extract estimates about $g_{\phi \gamma \gamma}$ and $m_{\phi}$, assuming equation (8.1) to be valid, when in principle, that may not be the case; therefore those estimates of the coupling constant and dilaton mass become questionable.

\section{Conclusion}

To conclude, in this work we have analysed the mixing pattern of dilatons with photons in a magnetized medium. Our analysis establishes that the mixing matrix for $\gamma \phi$ system is $3 \times 3$ not $2 \times 2$. We also found that, in a magnetized medium, the longitudinal DOF associated with the photon, doesn't get excited, by the dilatons; the same happens only for axions, as was rightly pointed out in 76. Using analytical techniques we have solved for the equations of motion of this system exactly. Following that we have estimated the strength of the polarimetric signals that is expected for a typical GRB geometry.

What we find is, there are regions in the parameter space over which, the signals are stable for some energy range, and there are regions for which the same may not be true ( for instance see figure 2, where the signal $\left(\Psi_{p}\right)$ is seen to stable over photon energy range $\left.1.0 \times 10^{-6} \mathrm{GeV}<\omega<1 \times 10^{-5} \mathrm{GeV}\right)$.

Given the fact that there are quite a few proposed satellite borne experiments in line [e.g. 82 [ 83$]$ [84] [85] [86], one needs to be careful while designing the detectors for them. The operational window of the energy range over which these detectors should work- may be decided after taking into account the lessons those emerged from the investigations like ours, in the respective simulations those usually considered for the purpose of detectors design.

Further more, the estimates of other observables like the spectral evolutions over energy, the magnitude of the fluence, the intensity of the spectrum in each polarization channel etc., those usually emerges from an analysis like ours, should also be taken into account - while designing the detectors ( i.e, their energy sensitivity, operational energy range, dead time fixation and physical dimension etc.). Once these are taken into account, it would help in getting better quality data - for understanding the nature of the ALPs and their parameters. 


\section{Note}

For more details on discrete symmetries of different mediums and their effects on propagation of EM signals, one can go through 87 and 88 .

\section{Acknowledgment}

The authors would like to thank Prof. Georg G. Raffelt of Max-Planck-Institut fur Physik for being kind enough to going through the manuscript and encouragements.

\section{Appendix}

\section{Polarimetry with magnetized media}

In this part of the work, we discuss the polarization effects on an electromagnetic field due to magnetized media. The description of photon propagation in a magnetized media are provided by the set of equations describing the evolution of the respective degrees of freedom, one of them having plane of polarization along the magnetic field called $A_{\|}$and the other one having plane of polarization orthogonal to the same. This set is given by

$$
\begin{aligned}
& \left(k^{2}-\Pi_{T}^{2}\right) A_{\|}(\omega, z)+i F A_{\perp}(\omega, z)=0, \\
& \left(k^{2}-\Pi_{T}^{2}\right) A_{\perp}(\omega, z)-i F A_{\|}(\omega, z)=0 .
\end{aligned}
$$

In equations (A1) and (A2), the strength of magnetization is carried by the variable $F$ and the same is given by $F=\frac{\omega_{p}^{2} e B \cos \theta}{\omega m_{e}}$. Plasma frequency is denoted by $\omega_{p}, \omega$ is the energy of photon, $e B$ is the magnetic field strength. Defining, $\Omega^{2}=\left(\frac{\omega_{p}^{2}}{2 \omega}-\omega\right)$. The eqns. (A1) and (A2) can further be written terms of a mixing matrix $\mathbf{M}$ causing mixing between the components of polarization $A_{\|}$and $A_{\perp}$, in the following fashion,

$$
i \partial_{z}\left[\left[\begin{array}{ll}
1 & 0 \\
0 & 1
\end{array}\right]+\left[\begin{array}{cc}
\Omega^{2} & -\frac{i F}{2 \omega} \\
-\frac{i F}{2 \omega} & \Omega^{2}
\end{array}\right]\right]\left[\begin{array}{l}
A_{\|}(\omega, z) \\
A_{\perp}(\omega, z)
\end{array}\right]=0 .
$$

To find the solutions of $A_{\|}$and $A_{\perp}$, we need eigen values and the eigen vectors for matrix M. Using the eigen vectors and eigen values $\lambda_{+}$and $\lambda_{-}$, the unitary matrices turn out to be,

$$
\tilde{U}=\frac{1}{\sqrt{2}}\left[\begin{array}{ll}
i & 1 \\
1 & i
\end{array}\right] \text { and, } \tilde{U}^{\dagger}=\frac{1}{\sqrt{2}}\left[\begin{array}{cc}
-i & 1 \\
1 & -i
\end{array}\right] .
$$

Using them, we finally arrive at the solutions of $A_{\|}$and $A_{\perp}$ in terms of the initial conditions $A_{\|}(\omega, 0)$ $A_{\perp}(\omega, 0)$ the solutions are,

$$
\begin{aligned}
A_{\|}(\omega, z) & =\left[\cos \left(\Omega^{2} z\right) \cos \left(\frac{F z}{2 \omega}\right) A_{\|}(\omega, 0)-\cos \left(\Omega^{2} z\right) \sin \left(\frac{F z}{2 \omega}\right) A_{\perp}(\omega, 0)\right] \\
& +i\left[\sin \left(\Omega^{2} z\right) \cos \left(\frac{F z}{2 \omega}\right) A_{\|}(\omega, 0)-\sin \left(\Omega^{2} z\right) \sin \left(\frac{F z}{2 \omega}\right) A_{\perp}(\omega, 0)\right],
\end{aligned}
$$




$$
\begin{aligned}
A_{\perp}(\omega, z) & =\left[\cos \left(\Omega^{2} z\right) \cos \left(\frac{F z}{2 \omega}\right) A_{\perp}(\omega, 0)+\cos \left(\Omega^{2} z\right) \sin \left(\frac{F z}{2 \omega}\right) A_{\|}(\omega, 0)\right] \\
& +i\left[\sin \left(\Omega^{2} z\right) \sin \left(\frac{F z}{2 \omega}\right) A_{\|}(\omega, 0)+\sin \left(\Omega^{2} z\right) \cos \left(\frac{F z}{2 \omega}\right) A_{\perp}(\omega, 0)\right] .
\end{aligned}
$$

It is easy to check the consistency of the solutions, by noting that for $F=0$, there is no mixing between $A_{\|}$and $A_{\perp}$. Now we make use of the solutions to obtain the Stokes parameters $I, Q, U$ and $V$ and following that can evaluate the polarization angle $\boldsymbol{\Psi}$ and ellipticity angle $\chi$ from them. The expressions for $I, Q, U$ and $V$ are given as follows,

$$
\begin{aligned}
I & =A_{\|}^{2}(\omega, 0)+A_{\perp}^{2}(\omega, 0), \\
Q & =\cos \left(\frac{F z}{\omega}\right)\left[A_{\|}^{2}(\omega, 0)-A_{\perp}^{2}(\omega, z)\right]-2 \sin \left(\frac{F z}{\omega}\right) A_{\perp}(\omega, 0) A_{\|}(\omega, 0), \\
U & =\sin \left(\frac{F z}{\omega}\right)\left[A_{\|}^{2}(\omega, 0)-A_{\perp}^{2}(\omega, 0)\right]+2 \cos \left(\frac{F z}{\omega}\right) A_{\perp}(\omega, 0) A_{\|}(\omega, 0) .
\end{aligned}
$$

It turns out that, in this case the Stokes parameter $V$ describing circular polarization is equal to zero. It is to be noted that the Stokes parameter $I$ is now independent of the path length $z$, which provides the consistency check of energy conservation of the system. Also, the rate of rotation of the plane of polarization, with change of path length distance, is proportional to the inverse square of energy of photon, i.e.,

$$
\frac{d \psi}{d z}=\frac{\omega_{p}^{2} e B \cos \theta}{2 \omega^{2} m_{e}} .
$$

The $\omega$ dependence of this result matches with the same reported in [67. Therefore one can state that the effect of magnetized medium alone on the polarimetric signature of light can be predicted by studying the state of circular polarization of light along with energy dependence of rate of rotation of the polarization angle per unit length by studying the system when the magnetic field $\vec{B}$ is along $\vec{k}$. On the other hand when the angle between $\vec{k}$ and $\vec{B}$ is $\frac{\pi}{2}$ then the effect of magnetized media of polarization of the electromagnetic beam vanishes and the variation of polarization with $\omega$ can be found in [45. Similar studies only for scalars or pseudoscalars have been performed in 62 , that show different outcome.

\section{References}

[1] Y. M. Cho and J. H. Kim. Phys. Rev. D 79, 023504 (2009).

[2] Thibault Damour and John F. Donoghue, Phys. Rev. D 82, 084033 (2010).

[3] Maurizo Gasperini, Elements Of String Cosmology, Cambridge, UK, (Cambridge University Press 2007)

[4] Bobby S. Acharya, Mansi Dhuria, Diptimoy Ghosh, Anshuman Maharana. JCAP 11, 035 (2019). 
[5] Claudio Corian', Luigi Delle Rose, Antonio Quintavalle and Mirko Serino, JHEP, 077,1306 (2013); W. D.Goldberger, B. Grinstein and W. Skiba Phys. Rev. Lett. 100, 111802 (2008).

[6] A. Salam and J.A. Strathdee, Phys. Rev. 184, 1760 (1969).

[7] Y.M. Cho, Phys. Rev. D 41, 2462 (1990).

[8] Y.M. Cho and J.H. Yoon, Phys. Rev. D 47, 3465 (1993).

[9] Y.M. Cho, in Proceedings of XXth Yamada Conference, edited by S. Hayakawa and K. Sato (University Academy,Tokyo 1988).

[10] Koijam Manihar Singh, Kangujam Priyo kumar Singh, Mod. Phys. Lett. A 34 09, 1950056 (2019).

[11] Bobby Samir Acharya, Anshuman Maharana, Francesco Muia. JHEP 03, 048 (2019).

[12] Dongjin Chway, arXiv:190109760 [gr-qc], kioon choi, Dongjin Chway, Chang Sub Shin, JHEP 142, 1811 (2018).

[13] T. Damour and J. F. Donoghue, Phys.Rev. D 82, 084033 (2010).

[14] L. Maiani, R. Petronzio and E. Zavattini. Phys. Lett. B 175, 359 (1986).

[15] G. Raffelt and L. Stodolsky. Phys. Rev. D 37, 1237 (1988).

[16] V. Baukh, A. Zhuk and T. Kahniashvili. Phys. Rev. D 76, 027502 (2007).

[17] A.K. Ganguly and R. Parthasarathy. Phys. Rev. D 68, 106005 (2003).

[18] M. Giannotti, I. Irastorza, J. Redondo and A. Ringwald. JCAP 05, 057 (2016).

[19] A. K. Ganguly and M. K. Jaiswal. Phys. Rev. D 90, 026002 (2014).

[20] H. Perez Rojas and A. E. Shabad. Ann. Phys. (N.Y.) 121, 432-455 (1979)

[21] J. C. DOlivo, J. F. Nieves and S. Sahu. Phys. Rev. D. 67 025018, (2003).

[22] J.R. Espinosa, C. Grojean, G. Panico, A. Pomarol, O. Pujolas, and G. Servant Phys. Rev. Lett. 115, $251803(2015)$. 
[23] S. A. Hoedl, F. Fleischer, E. G. Adelberger and B. R. Heckel. Phys. Rev. Lett. 106, 041801 (2011).

[24] Sonny Mantry, Mario Pitschmann and Michael J. Ramsey-Musolf. arXiv:1411.2162v1.

[25] P. Majumdar. Mod Phys. Lett. A 19, 1319(2004).

[26] G.G. Raffelt. Chicago Univ. Pr., Chicago U.S.A.(1996).

[27] A. Ringwald. PoS(NEUTEL2015)021 arXiv:1506.04259.

[28] M. Giannotti. arXiv:1508.07576.

[29] A. Ayala, I. Dominguez, M. Giannotti, A. Mirizzi and O. Straniero. Phys. Rev. Lett. 113, 191302 (2014).

[30] Ken’ichi Saikawa and Tsutomu T. Yanagida. arXiv:1907.07662v1.

[31] M. Giannotti, I. G. Irastorza, J. Redondo, A. Ringwaldd and K. Saikawad. JCAP 10, 010 (2017).

[32] Larry McLerran. J. Exp. Theor. Phys. 120 3, 376 (2015).

[33] R. Chanda, J. F. Nieves and P. B. Pal. Phys.Rev. D 37, 2714 (1988).

[34] K. Zioutas et al. (CAST Collaboration). Phys. Rev. Lett. 94,121301 (2005).

[35] K. Zioutas et al. (CAST Collaboration). Nat. Phys. 13, 584 (2017).

[36] E. Armengaud et al. JINST 9 T05002. arXiv:1401.3233 (2014).

[37] XENON100 collaboration, E. Aprile et al. Phys. Rev. D 90, 062009 (2014), [Erratum ibid.] D 95, 029904 (2017) arXiv:1404.1455.

[38] A. Caldwell, G. Dvali, B. Majorovits, A. Millar, G. Raffelt, J. Redondo, O. Reimann, F. Simon, F. Steffen (MADMAX Working Group), Phys. Rev. Lett. 118, 091801 (2017).

[39] DARWIN collaboration, J. Aalbers et al. JCAP 11, 017 (2016).

[40] I. G. Irastorza, J. Redondo. arXiv:1801.08127. 
[41] A. J. Millar, J. Redondo and F. D. Steffen. JCAP 10, 006 (2017)

[42] A. Kartavtsev, G. Raffelt, H. Vogel. JCAP 024, 01, 1701 (2017).

[43] S. Sarkar. Rep. Prog. Phys. 59, 12, 1493 (1996).

[44] Emi Masaki, Arata Aoki, Jiro Soda. Phys. Rev. D 96, 043519 (2017).

[45] Avijit K. Ganguly, Manoj K. Jaiswal. J. Korean Phys.Soc. 72, 1, 6 (2018).

[46] A. K. Ganguly, Ankur Chaubey, M. K. Jaiswal. Photon-Scalar Oscillation with Dim-5 operators: In a magnetizd media (to be communicated).

[47] E. W. Kolb, M. S. Turner, T. P. Walker Phys. Rev. D 34, 2197 (1986).

[48] A. D. Linde, Rep. Prog. Phys. 42, 389 (1979)

[49] E. s. Fradkin, Proc. Lebedev Phys. Inst. (1965)(English trans. 1967 by Consultants Bureau, New York).

[50] Jose F. Nieves, Palash B. Pal and David G. Unger, Phys.Rev.D 28908 (1983).

[51] Michel Le Bellac, Thermal field theory, Cambridge Monographs on Mathematical Physics.

[52] A. Hanson, T. Regge, C. Teitelboim, Academia Nazionale dei Lincei, Rome (1976).

[53] P.A.M Dirac, Lectures on Quantum mechanics, Yeshiva University Press, New York, 1964.

[54] E.C.G Sudarshan and N. Mukunda, John Wiley, New York (1974).

[55] J. Maharana, Pramana, 38, 5 (1992).

[56] S. L. Cherkas, K. G. Batrakov and D. Matsukevich Phys. Rev. D 66, 065011 (2002).

[57] J.D jackson. Third edition, Classical Electrodynamics, Wiley, (1998).

[58] C. Itzykson and J. B. Zuber, Quantum Field theory, Tata Mc Graw Hill (London), (1980). 
[59] S. Adler. Ann. Phys. 67, 599 (1971).

[60] A. K. Ganguly, P. Jain, and S. Mandal. Phys. Rev. D 79, 115014 (2009).

[61] N.V. Mikheev, G. Raffelt and L. A. Vassilevskaya Phys. Rev.D 58, 055008 (1998).

[62] R. Das, P.Jain and J. P. Ralston, R. Saha. Pramana 70, 439 (2008).

[63] A. K. Ganguly. Ann. Phys. 321, 6, 1457 (2006).

[64] H. Tercas, J. D. Rodrigues and J. T. Mendonca. Phys. Rev. Lett. 120, 181803 (2018).

[65] F. Miniati, G. Gregori, B. Reville and S. Sarkar. Phys. Rev. Lett. 121, 021301 (2018).

[66] K. Choi, H. Kim and T. Sekiguchi Phys. Rev. Lett. 121, 031102 (2018).

[67] A. K. Ganguly, S. Konar and P.B. Pal. Phys. Rev. D 60, 105014 (1999).

[68] B. Paczynski, ApJ. 308, L43 (1986).

[69] J. R. Ellis, N. E. Mavromatos, D.V. Nanopoulos and A. S. Sakharov, Astron. Astrophys 402, 409 (2003).

[70] A. Rubbia and A. Sakharov, Astroparticle Physics 29, 20 (2008).

[71] D. Yonetoku et. al. ApJ, 758, L1 (2012).

[72] K. Toma, S. Mukohyama, D. Yonetoku, T. Murakami, S. Gunji, T. Mihara, Y. Morihara, T. Sakashita, T. Takahashi, Y. Wakashima, H. Yonemochi, and N. Toukairin Phys. Rev. Lett. 109, $241104(2012)$.

[73] Eli Waxman Phys. Rev. Lett. 75, 3 (1995).

[74] https://hesperia.gsfc.nasa.gov/rhessi3/.

[75] https://www.nasa.gov/mission_pages/swift/main

[76] Matthew Lawson, Alexander J. Millar, Matteo Pancaldi, Edoardo Vitagliano, Frank Wilczek, Phys. Rev. Lett. 123, 14, 141802 (2019). 
[77] Granot, J., ApJ, 596, L17 (2003).

[78] Lyutikov, M., Pariev, V. I., \& Blandford, R., ApJ, 597, 998 (2003).

[79] Granot, J. Komissarov, S, S., \& Spitkovsky, A., MNRAS, 411,1323 (2012).

[80] Zhang, B., \& Yan, H., ApJ, 726, 90 (2011).

[81] Inoue, T., Asano, K., \& Ioka, K., ApJ, 734, 77 (2011).

[82] E. Silver, H. Schnopper. Ronaldo Belazzini, Enrico Costa, Giorgio Matt and Gianpiero Tagliaferri. Cambridge University Press, (2010).

[83] M. C. Weisskopf et al. RINP 6, 1179 (2016).

[84] Hooman Davoudiasl, Patrick Huber. Phys. Rev. Lett. 97,141302 (2006).

[85] B. Rani et al. arXiv:1903.04607v1. Z. Wadiasingh et al. arXiv:1903.05648.

[86] V. Tatischeff et al. arXiv:1706.07031v2.

[87] J. F. Nieves and P. B. Pal. Am. J. Phys. 62, 3 (1992).

[88] Palash B. Pal, Phys. Rev. D 102, 036004 (2020). arXiv:2005.09376

\section{Supplementary document}

\section{S13.1 Discrete symmetry effects on propagation}

We begin this section by defining the notations, used in this paper. We work in flat space with the metric tensor defined as $g^{\mu \nu}=g_{\mu \nu}=\operatorname{diag}(+1,-1,-1,-1)$. The contravariant and covariant four-vectors are defined as follows,

$$
x^{\alpha}=(t, \vec{x}), \quad x_{\alpha}=g_{\alpha \beta} x^{\beta}=(t,-\vec{x})^{T} .
$$

In our notation, the Greek indices are supposed to take values from 0 to 3 and the Latin ones between 1 to 3 .

The orthochronous improper Lorentz transformation, also called parity inversion transformation and non-orthochronous improper Lorentz transformation, also called the time reversal transformation, are defined by the matrices,

$$
\mathcal{P}^{\mu}{ }_{\nu}=\left(\mathcal{P}^{-1}\right)^{\mu}{ }_{\nu}=\left(\begin{array}{cccc}
1 & 0 & 0 & 0 \\
0 & -1 & 0 & 0 \\
0 & 0 & -1 & 0 \\
0 & 0 & 0 & -1
\end{array}\right) \quad \text { and } \quad \mathcal{T}^{\mu}{ }_{\nu}=\left(\mathcal{T}^{-1}\right)^{\mu}{ }_{\nu}=\left(\begin{array}{cccc}
-1 & 0 & 0 & 0 \\
0 & 1 & 0 & 0 \\
0 & 0 & 1 & 0 \\
0 & 0 & 0 & 1
\end{array}\right) \text {. }
$$


The corresponding unitary operators for parity and time reversal transformations, are identified as $U(P) \equiv \mathbf{P}$ and $U(T) \equiv \mathbf{T}$. Their action on any scalar $\Phi(x)$, generates the following transformation,

$$
\mathbf{P} \Phi(x) \mathbf{P}^{-1}=\Phi\left(\mathcal{P}^{-1} x\right) \text { and } \mathbf{T} \Phi(x) \mathbf{T}^{-1}=\Phi\left(\mathcal{T}^{-1} x\right) .
$$

Since $\mathbf{P}$ and $\mathbf{T}$ are their own inverse, therefore the right hand side of equation (ㅍ3), can also be represented in the following way,

$$
\mathbf{P} \Phi(x) \mathbf{P}^{-1}=\Phi(\mathcal{P} x) \text { and } \mathbf{T} \Phi(x) \mathbf{T}^{-1}=\Phi(\mathcal{T} x),
$$

for time reversal and parity inversion transformations.

The complex number $i=\sqrt{-1}$, under time reversal undergoes anti-unitary transformation, so that, the Hamiltonian $(H)$ a self adjoint operator, ( also represented by the zeroth component of the momentum four vector, $\left.k^{0}\right)$, retains the right sign under time $(\mathbf{T})$ reversal. However there are no such restriction for $\mathbf{P}$ and $\mathbf{T}$. So $i$ would remain inert, under them. Hence the transformation laws for $i$ are,

$$
\begin{aligned}
& \mathbf{T}(i) \mathbf{T}^{-1}=-i, \\
& \mathbf{P}(i) \mathbf{P}^{-1}=+i, \\
& \mathbf{C}(i) \mathbf{C}^{-1}=+i .
\end{aligned}
$$

Lastly, the totally anti-symmetric Levi-Civita tensor, defined as $\epsilon^{0123}=-\epsilon_{0123}=1$; picks up a -ve sign under both parity $(\mathbf{P})$ and time reversal $(\mathbf{T})$ transformations. That is, under parity transformation, it transforms as, $\mathbf{P} \epsilon^{\alpha \beta \gamma \sigma} \mathbf{P}^{-1}=-\epsilon^{\alpha \beta \gamma \sigma}=\epsilon_{\alpha \beta \gamma \sigma}$ and under time reversal, it transforms as, $\mathbf{T} \epsilon^{\alpha \beta \gamma \sigma} \mathbf{T}^{-1}=$ $-\epsilon^{\alpha \beta \gamma \sigma}=\epsilon_{\alpha \beta \gamma \sigma}$. In compact notation one can represent both of them as,

$$
\begin{aligned}
& \mathbf{T} \epsilon^{\alpha \beta \gamma \sigma} \mathbf{T}^{-1}=\epsilon_{\alpha \beta \gamma \sigma}, \\
& \mathbf{P} \epsilon^{\alpha \beta \gamma \sigma} \mathbf{P}^{-1}=\epsilon_{\alpha \beta \gamma \sigma}, \\
& \mathbf{C} \epsilon^{\alpha \beta \gamma \sigma} \mathbf{C}^{-1}=\epsilon^{\alpha \beta \gamma \sigma} .
\end{aligned}
$$

The last line follows from, the fact that, the net charge associated with any system remains the same under a duality transformation.

\section{S13.2 Discrete transformation properties of the fundamental vectors.}

Next we come to discuss the transformation properties of space time four vector $x^{\mu}$. Under time reversal $(\mathbf{T})$, space inversion $(\mathbf{P})$ and charge conjugation $(\mathbf{C})$ transformations, the four vector $x^{\mu}$ transforms as,

$$
\begin{aligned}
\mathbf{T} x^{\mu} \mathbf{T}^{-1} & =(-t, \vec{x})=-x_{\mu}, \\
\mathbf{P} x^{\mu} \mathbf{P}^{-1} & =(t,-\vec{x})=+x_{\mu}, \\
\mathbf{C} x^{\mu} \mathbf{C}^{-1} & =(t, \quad \vec{x})=+x^{\mu} .
\end{aligned}
$$

The last equation equation (S13) follows from the fact that, under charge conjugation the space-time 4 -vectors undergo no change.

In the light of equations ( $\mathbf{S 1 1}$ - (S13) $)$, we can discuss the transformation rules of the momentum 4 -vectors associated with the system. The same by the virtue of being observables, are supposed to be selfadjoint operators. Therefore following the rules of relativistic quantum theory, they can be represented by;

$$
\begin{aligned}
& k^{\mu} \equiv i \partial^{\mu}=i\left(\partial^{0}, \partial^{j}\right)=i \frac{\partial}{\partial x_{\mu}}=i\left(\partial_{0},-\nabla\right), \\
& k_{\mu} \equiv i \partial_{\mu}=i\left(\partial_{0}, \partial_{j}\right)=i \frac{\partial}{\partial x^{\mu}}=i\left(\partial_{0}, \quad \nabla\right) .
\end{aligned}
$$




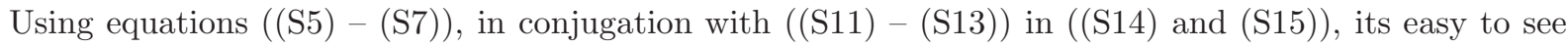
that, under the operation of $\mathbf{T}$ reversal transformation, $k^{\mu}$ transforms as; $\mathbf{T} k^{\mu} \mathbf{T}^{-1}=-i\left(-\partial^{0}, \partial^{j}\right)=$ $i\left(\partial^{0},-\partial^{j}\right)=i \partial_{\mu}=k_{\mu}$. Similarly, under $\mathbf{P}$, it transforms as $\mathbf{P} k^{\mu} \mathbf{P}^{-1}=i\left(\partial^{0},-\partial^{j}\right)=i\left(\partial_{0}, \partial_{j}\right)=i \partial_{\mu}=k_{\mu}$. However $k^{\mu}$ remains the same under $\mathbf{C}$ transformation. Hence, the transformation laws of $k^{\mu}$ individually under $\mathbf{T}, \mathbf{P}$ and $\mathbf{C}$ are given by,

$$
\begin{aligned}
\mathbf{T} k^{\mu} \mathbf{T}^{-1} & =k_{\mu} . \\
\mathbf{P} k^{\mu} \mathbf{P}^{-1} & =k_{\mu}, \\
\mathbf{C} k^{\mu} \mathbf{C}^{-1} & =k^{\mu},
\end{aligned}
$$

Finally, the centre of mass four velocity of the medium, defined as, $u^{\mu}=\frac{d x^{\mu}}{d \tau}$, when $d \tau=\sqrt{d t^{2}-d x^{2}}$ is the differential proper-time interval ), have the following transformation properties under time reversal, parity and charge conjugation transformations:

$$
\begin{aligned}
& \mathbf{T} u^{\mu} \mathbf{T}^{-1}=\left(-u^{0}, u^{j}\right)=-u_{\mu}, \\
& \mathbf{P} u^{\mu} \mathbf{P}^{-1}=\left(u^{0},-u^{j}\right)=+u_{\mu}, \\
& \mathbf{C} u^{\mu} \mathbf{C}^{-1}=\left(u^{0},-u^{j}\right)=-u^{\mu} .
\end{aligned}
$$

The last property (S21) follows from the observation, that the statistical part of the thermal propagator in real time thermal QFT should remain invariant under the operation of charge conjugation. The factor that carry the information about temperature and chemical potential of a fermionic quantum system according to quantum statistical field theory, is given by,

$$
\eta_{T}(p . u)=\left[\Theta(p . u) n_{F}(p . u, \mu, \beta)+\Theta(-p . u) n_{F}(-p . u,-\mu, \beta)\right],
$$

with $\Theta(p . u)$ the step function and $n_{F}(p . u, \mu, \beta)=\frac{1}{e^{\left(\frac{p \cdot u-\mu}{\beta}\right)}+1}$ the Fermi distribution function, and $\beta$ the inverse of temperature. One can see that under the operation of charge conjugation $(\mathbf{C})$ transformation, if $\mu \rightarrow-\mu$, then in order to have the statistical factor $\eta_{T}(p . u)$ inert under the same, one should have $u^{\mu} \rightarrow-u^{\mu}$ [67. As explained there ([67), the same in the rest frame of the medium, is given by $u^{\mu}=(1,0,0,0)$; for the remaining part of this paper we shall assume this to be true.

\section{S13.3 Discrete transformation properties of the fields}

Here we note down the transformation properties of four-vector potential $A^{\mu}\left(x^{\alpha}\right)$, followed by field strength tensor $F^{\mu \nu}\left(x^{\alpha}\right)$ under the set of discrete transformations (i.e., $\mathbf{C}, \mathbf{P}$ and $\mathbf{T}$ ). The transformation properties of $A^{\mu}\left(x^{\alpha}\right)$ following [58, under parity $\mathbf{P}$, time reversal $\mathbf{T}$ and charge conjugation $\mathbf{C}$ are:

$$
\begin{aligned}
& \mathbf{T} A^{\mu}\left(x^{\alpha}\right) \mathbf{T}^{-1}=+A_{\mu}\left(-x_{\alpha}\right), \\
& \mathbf{P} A^{\mu}\left(x^{\alpha}\right) \mathbf{P}^{-1}=+A_{\mu}\left(x_{\alpha}\right), \\
& \mathbf{C} A^{\mu}\left(x^{\alpha}\right) \mathbf{C}^{-1}=-A^{\mu}\left(x^{\alpha}\right) .
\end{aligned}
$$

Using the equations (S23) to (S25) and the transformation properties of $\partial_{\nu}$ under $\mathbf{C}, \mathbf{P}$ and $\mathbf{T}$, the transformation properties of the field strength tensor $F^{\mu \nu}(x)$, under them can be found. And they happen to be:

$$
\begin{aligned}
& \mathbf{T} F^{\mu \nu}\left(x^{\alpha}\right) \mathbf{T}^{-1}=-F_{\mu \nu}\left(-x_{\alpha}\right), \\
& \mathbf{P} F^{\mu \nu}\left(x^{\alpha}\right) \mathbf{P}^{-1}=+F_{\mu \nu}\left(x_{\alpha}\right), \\
& \mathbf{C} F^{\mu \nu}\left(x^{\alpha}\right) \mathbf{C}^{-1}=-F^{\mu \nu}\left(x^{\alpha}\right) .
\end{aligned}
$$


Finally, the relation between the electric or magnetic field components and the components of the field strength tensor, $F^{\mu \nu}$ are given by,

$$
F^{\mu \nu}=\left(\begin{array}{cccc}
0 & -E_{x} & -E_{y} & -E_{z} \\
E_{x} & 0 & -B_{z} & B_{y} \\
E_{y} & B_{z} & 0 & -B_{x} \\
E_{z} & -B_{y} & B_{x} & 0
\end{array}\right) \quad \text { and } \quad F_{\mu \nu}=g_{\mu \alpha} F^{\alpha \beta} g_{\beta \nu}=\left(\begin{array}{cccc}
0 & E_{x} & E_{y} & E_{z} \\
-E_{x} & 0 & -B_{z} & B_{y} \\
-E_{y} & B_{z} & 0 & -B_{x} \\
-E_{z} & -B_{y} & B_{x} & 0
\end{array}\right) \text {. }
$$

Using the relation given by (S29) and ( $(\underline{\mathbf{S 2 6}})-(\underline{\mathbf{S 2 8}})$ ) one can establish the $\mathbf{C}, \mathbf{P}$ and $\mathbf{T}$ transformation properties of the electric $E$ and magnetic $B$ fields. We have performed the same and our transformation properties obtained this way matches with that of Jackson [57]

\section{S13.3.1 C, P and T transformations properties of the basis vectors :}

We next find out the $\mathbf{C}, \mathbf{P}$, and $\mathbf{T}$ transformation properties of the basis vectors. We start with vector $b^{(1) \mu}$, given by $b^{(1) \mu}=k_{\nu} F^{\mu \nu}$. Using the transformation properties of $k_{\nu}$ and $F^{\mu \nu}$ under $\mathbf{P}, \mathbf{T}$ and $\mathbf{C}$, one can show that:

$$
\begin{aligned}
& \mathbf{T} b^{(1) \mu} \mathbf{T}^{-1}=-b_{\mu}^{(1)}, \\
& \mathbf{P} b^{(1) \mu} \mathbf{P}^{-1}=+b_{\mu}^{(1)} \\
& \mathbf{C} b^{(1) \mu} \mathbf{C}^{-1}=-b^{(1) \mu} .
\end{aligned}
$$

Similarly using the definition of the 4 -vector, $b^{(2) \mu}$, as $b^{(2) \mu}=\frac{1}{2} k_{\beta} \bar{F}_{\lambda \rho} \epsilon^{\beta \lambda \rho \mu}$ one can show the transformations of the same under $\mathbf{T}, \mathbf{P}$ and $\mathbf{C}$. They happen to be,

$$
\begin{aligned}
\mathbf{T} b^{(2) \mu} \mathbf{T}^{-1} & =-b_{\mu}^{(2)} \\
\mathbf{P} b^{(2) \mu} \mathbf{P}^{-1} & =+b_{\mu}^{(2)} \\
\mathbf{C} b^{(2) \mu} \mathbf{C}^{-1} & =-b^{(2) \mu}
\end{aligned}
$$

Next we are left with estimating the same transformation rules for four vectors $I^{\mu}$ and $\tilde{u}^{\mu}$. But before we perform that, we need to have the transformations of, $b^{(2)} \cdot \tilde{u}$ and $k \cdot u$ under $\mathbf{C}, \mathbf{P}$ and $\mathbf{T}$. The dot products in $b^{(2)} \cdot \tilde{u}$ and $k \cdot u$ are to be understood as dot products in 4-dimensions. The transformations for $b^{(2)} \cdot \tilde{u}$ are,

$$
\begin{aligned}
& \mathbf{T}\left(\tilde{u} \cdot b^{(2)}\right) \mathbf{T}^{-1}=\left(\tilde{u} \cdot b^{(2)}\right), \\
& \mathbf{P}\left(\tilde{u} \cdot b^{(2)}\right) \mathbf{P}^{-1}=\left(\tilde{u} \cdot b^{(2)}\right), \\
& \mathbf{C}\left(\tilde{u} \cdot b^{(2)}\right) \mathbf{C}^{-1}=\left(\tilde{u} \cdot b^{(2)}\right) .
\end{aligned}
$$

Similarly, follows the transformation laws for the four dimensional dot product $k \cdot u$, from the individual transformation laws of $k^{\mu}$ and $u_{\mu}$, and they are,

$$
\begin{aligned}
& \mathbf{T}(u \cdot k) \mathbf{T}^{-1}=-(u \cdot k), \\
& \mathbf{P}(u \cdot k) \mathbf{P}^{-1}=+(u \cdot k), \\
& \mathbf{C}(u \cdot k) \mathbf{C}^{-1}=-(u \cdot k) .
\end{aligned}
$$

Recalling, the four vector $I^{\nu}$, to be given by

$$
I^{\nu}=\left(b^{(2)^{\nu}}-\frac{\left(\tilde{u}^{\mu} b_{\mu}^{(2)}\right)}{\tilde{u}^{2}} \tilde{u}^{\nu}\right) ;
$$


the transformation laws for, $I^{\mu}$, using the transformation laws for equation (S38) turns out to be,

$$
\begin{aligned}
\mathbf{T} I^{\mu} \mathbf{T}^{-1} & =-I_{\mu}, \\
\mathbf{P} I^{\mu} \mathbf{P}^{-1} & =+I_{\mu}, \\
\mathbf{C} I^{\mu} \mathbf{C}^{-1} & =-I^{\mu} .
\end{aligned}
$$

Lastly the same for $\tilde{u}^{\mu}$, using equation (S41) are found to be,

$$
\begin{aligned}
\mathbf{T} \tilde{u}^{\mu} \mathbf{T}^{-1} & =-\tilde{u}_{\mu}, \\
\mathbf{P} \tilde{u}^{\mu} \mathbf{P}^{-1} & =+\tilde{u}_{\mu}, \\
\mathbf{C} \tilde{u}^{\mu} \mathbf{C}^{-1} & =-\tilde{u}^{\mu} .
\end{aligned}
$$

\section{S13.4 Discrete transformation properties: EM form-factors}

The EM form-factors associated with the gauge potential, $A_{\mu}$, are $A_{\|}, A_{\perp}, A_{L}$ would have their own transformation laws under $\mathbf{C}, \mathbf{P}$ and $\mathbf{T}$. In the next few lines we would outline the detail of finding their transformation rule. The basic principle lies with finding out the transformation rules for the time-like and the space like components of $A_{\mu}$, following from equations (S23) to (S25). We recall the following transformations, that follows from, equations (S23) to (S25),

$$
\begin{aligned}
& \mathbf{T} A^{0} \mathbf{T}^{-1}=+A^{0}, \quad \mathbf{P} A^{0} \mathbf{P}^{-1}=+A^{0}, \quad \mathbf{C} A^{0} \mathbf{C}^{-1}=-A^{0}, \\
& \mathbf{T} A^{i} \mathbf{T}^{-1}=-A^{i}, \quad \mathbf{P} A^{i} \mathbf{P}^{-1}=-A^{i}, \quad \mathbf{C} A^{i} \mathbf{C}^{-1}=-A^{i} .
\end{aligned}
$$

Next we can express the time-like and the space-like parts of the gauge potentials in terms of the EM form-factor from the definition of the gauge potential;

$$
A^{\alpha}(k)=A_{\|}(k) \mathrm{N}_{1} \mathrm{~b}^{(1) \alpha}+\mathrm{A}_{\perp}(\mathrm{k}) \mathrm{N}_{2} \mathrm{I}^{\alpha}+\mathrm{A}_{\mathrm{L}}(\mathrm{k}) \mathrm{N}_{\mathrm{L}} \tilde{\mathrm{u}}^{\alpha} .
$$

They turn out to be,

$$
\begin{aligned}
A^{0} & =N_{1} b^{(1) 0} A_{\|}+N_{2} I^{0} A_{\perp}+N_{L} \tilde{u}^{0} A_{L}, \\
A^{i} & =N_{1} b^{(1) i} A_{\|}+N_{2} I^{i} A_{\perp}+N_{L} \tilde{u}^{i} A_{L} .
\end{aligned}
$$

If we use the relations given by equation (S48) on equations (S50) and (S51), we would arrive at,

$$
\begin{aligned}
& \mathbf{T} A_{\|} \mathbf{T}^{-1}=-A_{\|}, \mathbf{T} A_{\perp} \mathbf{T}^{-1}=-A_{\perp}, \mathbf{T} A_{L} \mathbf{T}^{-1}=-A_{L}, \\
& \mathbf{P} A_{\|} \mathbf{P}^{-1}=+A_{\|}, \mathbf{P} A_{\perp} \mathbf{P}^{-1}=+A_{\perp}, \mathbf{P} A_{L} \mathbf{P}^{-1}=+A_{L}, \\
& \mathbf{C} A_{\|} \mathbf{C}^{-1}=+A_{\|}, \mathbf{C} A_{\perp} \mathbf{C}^{-1}=+A_{\perp}, \quad \mathbf{C} A_{L} \mathbf{C}^{-1}=+A_{L} .
\end{aligned}
$$

once we take the transformation laws of the basis vectors under $\mathbf{T}, \mathbf{P}$ and $\mathbf{C}$ into account.

\section{S13.5 The photon polarization tensor in magnetized media.}

The photon polarization tensor, $\Pi_{\mu \nu}^{p}(k)$, in a magnetized medium to $O(e B)$, can be expressed parametrically in the following form,

$$
\Pi_{\mu \nu}^{p}(k)=\Pi^{p}(k) \frac{i}{\sqrt{(k u)^{2}-k^{2}}} \epsilon_{\mu \nu \beta_{\|} \delta} u^{\tilde{\beta}_{\|}} k^{\delta} .
$$

The notations being, in the Levi-Civita tensor $\epsilon_{\mu \nu \beta_{\|} \delta}$, the subscripted index $\beta_{\|}$is allowed to take only two values, either 0 or 3 ; although the subscripted index $\tilde{\beta}_{\|}$is also allowed to take only those two numerical 
values, but there is a difference; the difference being, when $\beta_{\|}=0$ then $\tilde{\beta}_{\|}=3$ and when $\beta_{\|}=3$ then $\tilde{\beta}_{\|}=0$.

This leaves us with fixing the functional form of the argument of the scalar EM form-factor $\Pi^{p}(k)$. The functional form of the arguments have to be Lorentz scalar. Though, in principle these Lorentz scalars can be of the form: $u . k$ or $k^{2}$ etc., however their power and functional-structure would be decided on the basis of some general Quantum Field Theoretic arguments; that a two point function is supposed to follow.

One of these formal Quantum Field Theoretic arguments is, that the partition function or S matrix should be unitary: this requirement dictates that the two point function (photon polarization tensor) must be hermitian i.e.,

$$
\Pi_{\mu \nu}^{p}(k)=\Pi_{\nu \mu}^{p *}(k) .
$$

It should obey Bose symmetry, i.e.,

$$
\Pi_{\mu \nu}^{p}(k)=\Pi_{\nu \mu}^{p}(-k) .
$$

Additionally, since the same is being evaluated for a theory that is CPT invariant, the polarization tensor should also remain invariant under the combined operation of CPT transformation, as well as- invariant separately- under $\mathbf{C}$ and $\mathbf{P T}$. That is:

$$
(\mathbf{C P T}) \Pi_{\mu \nu}^{p}(k)(\mathbf{C P T})^{-1}=\Pi_{\mu \nu}^{p}(k), \mathbf{C} \Pi_{\mu \nu}^{p}(k) \mathbf{C}^{-1}=\Pi_{\mu \nu}^{p}(k) \quad \& \quad(\mathbf{P T}) \Pi_{\mu \nu}^{p}(k)(\mathbf{P T})^{-1}=\Pi_{\mu \nu}^{p}(k)
$$

Fulfilment of the condition of hermiticity given by equation (S56), is satisfied due to the presence of the Levi-Civita symbol along with the multiplicative factor of $i(i . e ., \sqrt{(-1)})$, below the pair production threshold.

Similarly, the condition of Bose symmetry given by equation (S57), is fulfilled due to the presence of the same Levi-Civita symbol multiplied by four vector $k$. This however puts strong restriction on the functional form of form-factor $\Pi^{p}(k)$, that is, it just tells us that, the same has to be even function of the scalars like $k^{2}$ or $k . u$ etc. Evaluation of the same from the corresponding one loop Feynman diagram -using perturbation theory shows - that, it indeed is a function of $\sqrt{(k \cdot u)^{2}}=\omega$.

The Charge conjugation symmetry, similarly dictates that: since the tensorial structure is odd under $\mathbf{C}$ therefore to maintain overall $\mathbf{C}$ invariance the form-factor $\Pi^{p}(k)$ should be either odd in B or odd in chemical potential $\mu$, but not both. Since we have evaluated this term to order $e B$ and that too as a scalar in $B$, hence it can be utmost be a function of $\sqrt{B^{2}}$. Hence it should be odd in chemical potential. The same is achieved automatically, if the statistical factor is composed of the difference between electron and positron distribution functions $\left(n_{e^{ \pm}}(p . u, \mu, \beta)\right)$. That is with the presence of a piece that represent the difference in the fermionic densities, like $\left(n_{\left(e^{-}\right)}-n_{\left(e^{+}\right)}\right)$.

Having discussed the structure of the form-factor on general grounds, we now provide with the expressions of the form-factor $\Pi^{p}(k)$ as obtained from analytical evaluation of the same carried out in 67 vi-a-vis the same obtained on the ground of the general analysis presented above. The same turns out to be,

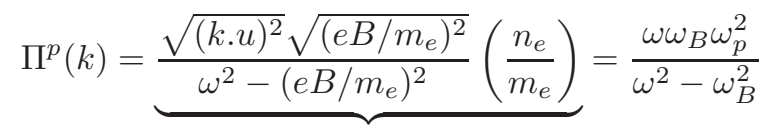

In the expression above, given by equation (S59), $\omega_{B}=\frac{e B}{m_{e}}$ also called the Larmor frequency and $\omega_{p}$, 
is the plasma frequency, given by $\omega_{p}=\sqrt{\frac{4 \pi \alpha n_{\left(e^{-}\right)}}{m_{e}}}$. The expression at the middle of eqn. (S59) (with an underbrace), represents the structure of $\Pi^{p}(k)$, as anticipated on the basis of our general arguments. And the term next to it, on the right hand side of the equal to sign, represents the same obtained after evaluating the one-loop-Feynman diagram. We conclude this section with the observation that, the EM form-factor $\Pi^{p}(k)$, appearing from the self energy correction due to magnetized matter effects, is $\mathbf{C}$ odd ( due to $\omega_{p}^{2} \propto n_{e^{-}}$) but PT even.

\section{S13.6 Discrete transformations of EOM}

The equation of motions for scalar-photon $(\phi \gamma)$ system are as follows;

$$
\begin{aligned}
\left(k^{2}-\Pi_{T}\right) A_{\|}(k)+i \Pi^{p}(k) N_{1} N_{2}\left[\epsilon_{\mu \nu \delta \beta} \frac{k^{\beta}}{|k|} u^{\tilde{\delta}_{\|}} b^{(1) \mu} I^{\nu}\right] A_{\perp}(k) & =\frac{i g_{\phi \gamma \gamma} \phi(k)}{N_{1}}, \\
\left(k^{2}-\Pi_{T}\right) A_{\perp}(k)-i \Pi^{p}(k) N_{1} N_{2}\left[\epsilon_{\mu \nu \delta \beta} \frac{k^{\beta}}{|k|} u^{\tilde{\delta}_{\|}} b^{(1) \mu} I^{\nu}\right] A_{\|}(k) & =0, \\
\left(k^{2}-\Pi_{L}\right) A_{L}(k) & =0, \\
\left(k^{2}-m^{2}\right) \phi(k) & =-\frac{i g_{\phi \gamma \gamma} A_{\|}(k)}{N_{1}} .
\end{aligned}
$$

An useful exercise towards establishing the correctness of the set of equations of motions, is to show that, these equations obey some symmetry condition. The symmetry being the PT symmetry here. The first step towards establishing that, is to neglect $\Pi^{p}(k)$ in equations $\mathbf{S 6 0}-\mathbf{S 6 3}$. One can see that, after this exercise, only equations (S60) and (S63) remain coupled and the rest gets decoupled. This is expected, because, in the tree level interaction Lagrangian $\left(L_{I}=\frac{1}{4} \phi \bar{F}_{\mu \nu} f^{\mu \nu}\right)$, the scalar $\phi$ has no coupling with either $A_{L}$ or $A_{\perp}$. Using the decomposition of the vector potential in terms of the basis vectors,

$$
A^{\nu}(k)=A_{\|}(k) \mathrm{N}_{1} \mathrm{~b}^{(1) \nu}+\mathrm{A}_{\perp}(\mathrm{k}) \mathrm{N}_{2} \mathrm{I}^{\nu}+\mathrm{A}_{\mathrm{L}}(\mathrm{k}) \mathrm{N}_{\mathrm{L}} \tilde{\mathrm{u}}^{\nu}+\mathrm{N}_{\mathrm{k}} \mathrm{A}_{\mathrm{gf}}(\mathrm{k}) \mathrm{k}^{\nu}
$$

the issues related to coupling of various form factors, can be verified, by substituting (S64) in,

$$
L_{I}=\frac{1}{4} \phi \bar{F}_{\mu \nu} f^{\mu \nu}
$$

That is, substituting (S64) in the expression of the dynamical part of the photon field strength tensor, $f_{\mu \nu}$ in (S65). This also turns out to be true if effective Lagrangian with istropic self-energy tensor, $A_{\mu} \Pi^{\mu \nu}(T, \mu) A_{\nu}$ along with magnetic field induced tree level Lagrangian given by (S65) effects are considered.

Now operating $\mathbf{T}$ or PT on both sides of (S60) and $(\mathbf{S 6 3})$ one can convince one self , that the change in sign due to the discrete transformations are compensated with-out changing the equation structures. That is the equations remain invariant. To proof of the same when $\Pi^{p}(k)$ is retained, would require the transformation properties of the quantities (under $\mathbf{T}, \mathbf{C}, \mathbf{P}$ ) provided below. 


$$
\begin{aligned}
(\mathbf{P T}) A_{\|}(\mathbf{P T})^{-1} & =-A_{\|}, \\
(\mathbf{P T}) A_{\perp}(\mathbf{P T})^{-1} & =-A_{\perp}, \\
(\mathbf{P T}) i(\mathbf{P T})^{-1} & =-i, \\
(\mathbf{P T}) k^{\beta}(\mathbf{P T})^{-1} & =+k^{\beta}, \\
\left.(\mathbf{P T}) u^{\delta_{\|}} \mathbf{( P T}\right)^{-1} & =-u^{\delta_{\|}}, \\
(\mathbf{P T}) b^{(1) \mu}(\mathbf{P T})^{-1} & =-b^{(1) \mu}, \\
(\mathbf{P T}) I^{\nu}(P T)^{-1} & =-I^{\nu}, \\
(\mathbf{P T}) \epsilon_{\mu \nu \delta \beta}(\mathbf{P T})^{-1} & =\epsilon_{\mu \nu \delta \beta}, \\
(\mathbf{P T}) \Pi^{p}(k)(\mathbf{P T})^{-1} & =\Pi^{p}(k) .
\end{aligned}
$$

One can see that, if the equations (S60) - (S63), are multiplied from left by (PT) and from right by $(\mathbf{P T})^{-1}$ they remain invariant. That establishes that the equations are (PT) symmetric.

The form-factor corresponding to the longitudinal DOF $A_{L}$, still remains decoupled and, this DOF of photon propagates freely. This happens because, the scalar field $\phi$ and $A_{L}$ has no interaction at the tree level of the interaction Lagrangian.

Next we come to the structure of the mixing matrix. It should be noted that in absence of scalar photon interaction and $\Pi^{p}(k)$, the normal modes of the system are given by the three DOF, the transverse DOF $A_{\|}, A_{\perp}$ and the longitudinal DOF $A_{L}$. Such a system will be described by a $3 \times 3$ matrix, where the only the diagonal elements are nonzero. Upon the inclusion of magnetized matter effects the two transverse DOF $A_{\|}, A_{\perp}$ get coupled to each other, thus generating only five nonzero elements for the mixing matrix that is supposed to have nine elements . As one the includes the $\phi \gamma$ interaction, $A_{\|}$ gets coupled to $\phi$ and vice-versa, thus generating two more terms for the $3 \times 3$ mixing matrix, lifting the non-zero element count for the mixing matrix to seven. This can be verified from the explicit expression of the matrix. So the $3 \times 3$ mixing matrix has seven nonzero elements out of nine.

Note: After this paper was submitted for publication, we came across the reference [88, that deals with some of the issues those are discussed in this work. 